\title{
EXISTENCE, UNIQUENESS AND COALESCENCE OF DIRECTED PLANAR GEODESICS: PROOF VIA THE INCREMENT-STATIONARY GROWTH PROCESS
}

\author{
TIMO SEPP ̈̈LÄINEN
}

\begin{abstract}
We present a proof of the almost sure existence, uniqueness and coalescence of directed semi-infinite geodesics in planar growth models that is based on properties of an increment-stationary version of the growth process. The argument is developed in the context of the exponential corner growth model. It uses coupling, planar monotonicity, and properties of the stationary growth process to derive the existence of Busemann functions, which in turn control geodesics. This soft approach is in some situations an alternative to the much-applied 20-year-old arguments of C. Newman and co-authors. Along the way we derive some related results such as the distributional equality of the directed geodesic tree and its dual, originally due to L. Pimentel.
\end{abstract}

\section{INTRODUCTION}

1.1. The corner growth model and its geodesics. The setting for the planar corner growth model (CGM) with exponential weights is the following. $(\Omega, \mathfrak{S}, \mathbb{P}, \Theta)$ is a measure-preserving $\mathbb{Z}^{2}$ dynamical system. This means that $(\Omega, \mathfrak{S}, \mathbb{P})$ is a probability space and $\Theta=\left(\theta_{x}\right)_{x \in \mathbb{Z}^{2}}$ is a group of measurable bijections that acts on $\Omega$ and preserves $\mathbb{P}: \mathbb{P}\left(\theta_{x} A\right)=\mathbb{P}(A)$ for all events $A \in \mathfrak{S}$ and $x \in \mathbb{Z}^{2}$. The generic sample point of $\Omega$ is denoted by $\omega$. The random weights $Y=\left(Y_{x}\right)_{x \in \mathbb{Z}^{2}}$ are independent, identically distributed (i.i.d.) rate 1 exponentially distributed random variables on $\Omega$ that satisfy $Y_{x}(\omega)=Y_{0}\left(\theta_{x} \omega\right)$ for each $x \in \mathbb{Z}^{2}$ and almost every $\omega \in \Omega$.

The canonical choice is the product space $\Omega=\mathbb{R}_{\geqslant 0}^{\mathbb{Z}^{2}}$ with translations $\left(\theta_{x} \omega\right)_{y}=\omega_{x+y}$, an i.i.d. product measure $\mathbb{P}$ and the coordinate process $Y_{x}(\omega)=\omega_{x}$.

The last-passage percolation (LPP) process $G=G^{Y}$ is defined for $x \leqslant y$ (coordinatewise order) on $\mathbb{Z}^{2}$ by

$$
G_{x, y}=G(x, y)=\max _{x, \in \Pi_{x, y}} \sum_{k=0}^{|y-x|_{1}} Y_{x_{k}} .
$$

$\Pi_{x, y}$ is the set of up-right paths $x_{\bullet}=\left(x_{k}\right)_{k=0}^{n}$ that start at $x_{0}=x$ and end at $x_{n}=y$, with $n=|y-x|_{1}$. By definition, the increments of an up-right path satisfy $x_{k+1}-x_{k} \in\left\{\mathbf{e}_{1}, \mathbf{e}_{2}\right\}$. A path can be equivalently characterized in terms of its vertices or its edges. Both points of view are useful. See Figure 1.1 for an illustration. The zero-length path case is $G_{x, x}=\omega_{x}$. Our convention is that

$$
G_{x, y}=-\infty \quad \text { if } x \leqslant y \text { fails. }
$$

The shape function of the exponential CGM has been known since the seminal paper of Rost [18]:

$$
g(\xi)=\left(\sqrt{\xi}_{1}+\sqrt{\xi}_{2}\right)^{2} \quad \text { for } \xi=\left(\xi_{1}, \xi_{2}\right) \in \mathbb{R}_{\geqslant 0}^{2} .
$$

Date: Tuesday $16^{\text {th }}$ July, 2019.

2000 Mathematics Subject Classification. 60K35, 65K37.

Key words and phrases. Busemann function, coalescence, cocycle, competition interface, corner growth model, directed percolation, geodesic, last-passage percolation.

The author was partially supported by National Science Foundation grants DMS-1602486 and DMS-1854619 and by the Wisconsin Alumni Research Foundation. 


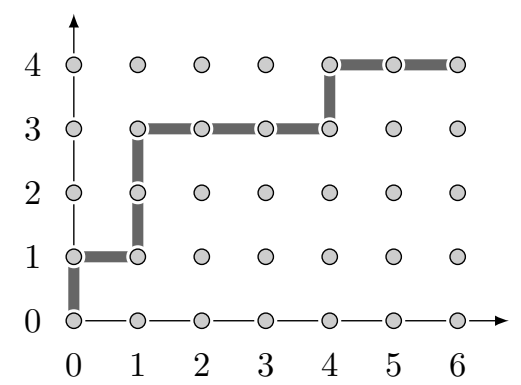

Figure 1.1. An example of an up-right path from $(0,0)$ to $(6,4)$ on the lattice $\mathbb{Z}^{2}$.

The shape theorem is the law of large numbers of the LPP process, uniform in all directions (Theorem 5.1 in [15], Theorem 3.5 in [19]):

TheOREm 1.1. Given $\varepsilon>0$, there exists a $\mathbb{P}$-almost surely finite random variable $K$ such that

$$
\left|G_{0, x}-g(x)\right| \leqslant \varepsilon|x|_{1} \quad \text { for all } x \in \mathbb{Z}_{\geqslant 0}^{2} \text { such that }|x|_{1} \geqslant K .
$$

An up-right path $\left(x_{i}\right)_{i \in I}$ indexed by a finite or infinite subinterval $I \subset \mathbb{Z}$ is a geodesic if it is the maximizing path between any two of its points:

$$
G_{x_{k}, x_{\ell}}=\sum_{i=k}^{\ell} Y_{x_{i}} \quad \text { for all } k<\ell \text { in } I .
$$

Since the weight distribution is continuous, maximizing paths between any two points are unique $\mathbb{P}_{-}$ almost surely. A geodesic $\left(x_{i}\right)_{i \in \mathbb{Z}_{\geqslant 0}}$ indexed by nonnegative integers is called a semi-infinite geodesic started at $x_{0}$, and a geodesic $\left(x_{i}\right)_{i \in \mathbb{Z}}$ indexed by the entire integer line is a bi-infinite geodesic. A semi-infinite or bi-infinite geodesic $x$. is $\mathbf{u}$-directed if $x_{n} / n \rightarrow \mathbf{u}$ as $n \rightarrow \infty$.

1.2. The purpose of the paper and its relation to past work. We address the existence, uniqueness and coalescence of semi-infinite geodesics in a given direction $\mathbf{u}$. The results themselves are not new. The purpose is to present an alternative proof of these known results.

Already for about two decades, geodesics and the closely related Busemann functions have been important in the study of first- and last-passage growth models, and recently also in positivetemperature polymer models. Proof techniques for the existence, uniqueness and coalescence of semi-infinite directed geodesics developed by C. Newman and co-authors $[13,14,16]$ have played a central role in this work. This approach controls the wandering of geodesics with estimates that rely on assumptions on the limit shape, to show that each direction has a geodesic and each geodesic has a direction. Almost sure coalescence is shown by a modification argument followed by a Burton-Keane type lack of space argument.

These techniques have been applied to great benefit in many models where sufficient solvability or symmetries enable the verification of the hypotheses imposed on the limit shape. In the exponential CGM this proof was implemented by P. A. Ferrari and L. Pimentel [10]. Examples of applications to LPP and positive-temperature polymers with quadratic limit shapes appear in $[1,2,4]$.

The proof developed in this paper replaces the estimates that control geodesics and the technical modification arguments with a softer proof that comes from structural properties. This proof can be substituted for Newman's proof in cases where sufficiently tractable increment-stationary versions of the growth process can be constructed. This may be possible in some situations where shiftinvariance and curvature are not available. This would be the case for example in models with inhomogeneous parameters, such as those whose limit shapes are studied in [8]. 
As a consequence of our development we establish Pimentel's distributional equality [17] of the directed geodesic tree and its dual, without recourse to mappings between the CGM and the totally asymmetric simple exclusion process (TASEP). It is useful to develop a proof of this result within the context of the growth model itself, for the purpose of extension to growth models and polymer models that are not connected to particle systems. Pimentel [17] used this duality to derive bounds on coalescence times.

1.3. Other related work. Recent work where coalescence of geodesics figures prominently include [11] on the CGM with general weights and $[6,7]$ on undirected first-passage percolation. These papers prove coalescence with the Licea-Newman argument. The proof given here does not presently apply to the models studied there because the properties of their Busemann functions are not yet sufficiently well understood.

Chaika and Krishnan [5] consider paths on a lattice defined by an ergodic field of nearest-neighbor "arrows", or local gradients. They use ergodicity and a very general volume argument to show that if coalescence fails, bi-infinite paths exist. Theirs would be an alternative proof of the (iii) $\Longrightarrow(\mathrm{i})$ implication for Busemann geodesics in Lemma 4.6 below. Our argument is more model-specific and uses the equal distribution of Busemann geodesics and their duals.

1.4. Notation and conventions. Points $x=\left(x_{1}, x_{2}\right), y=\left(y_{1}, y_{2}\right) \in \mathbb{R}^{2}$ are ordered coordinatewise: $x \leqslant y$ iff $x_{1} \leqslant y_{1}$ and $x_{2} \leqslant y_{2}$. The $\ell^{1}$ norm is $|x|_{1}=\left|x_{1}\right|+\left|x_{2}\right|$. A path as a sequence of points $\left(x_{k}\right)_{k=0}^{n}$ can be denoted by $x$. or by $x_{0, n}$. Subscripts indicate restricted subsets of the reals and integers: for example $\mathbb{Z}_{>0}=\{1,2,3, \ldots\}$ and $\mathbb{Z}_{>0}^{2}=\left(\mathbb{Z}_{>0}\right)^{2}$ is the positive first quadrant of the planar integer lattice. Boldface notation for special vectors: $\mathbf{e}_{1}=(1,0), \mathbf{e}_{2}=(0,1)$, and members of the simplex $\mathcal{U}=\left\{t \mathbf{e}_{1}+(1-t) \mathbf{e}_{2}: 0 \leqslant t \leqslant 1\right\}$ are denoted by $\mathbf{u}, \mathbf{v}$ and $\mathbf{w}$. For $0<\alpha<\infty$, $X \sim \operatorname{Exp}(\alpha)$ means that random variable $X$ has exponential distribution with rate $\alpha$, in other words $P(X>t)=e^{-\alpha t}$ for $t>0$ and $E(X)=\alpha^{-1}$. Functional arguments can be equivalently written as subscripts, as in $B(x, y, \omega)=B_{x, y}(\omega)$.

1.5. Acknowledgements. This paper benefited from numerous discussions and collaborations over the years, especially with E. Emrah, N. Georgiou, C. Janjigian, A. Krishnan, F. Rassoul-Agha, and A. Yılmaz. The exposition was improved by four anonymous referees.

\section{Main Results on DiRECTED SEMI-INFinite GeOdesics}

Here is a restatement of the assumption:

$(\Omega, \mathfrak{S}, \mathbb{P}, \Theta)$ is a measure-preserving $\mathbb{Z}^{2}$-dynamical system and $Y=\left(Y_{x}\right)_{x \in \mathbb{Z}^{2}}$ are i.i.d. $\operatorname{Exp}(1)$ random variables on $\Omega$ that satisfy $Y_{x}(\omega)=Y_{0}\left(\theta_{x} \omega\right) \mathbb{P}$-a.s.

The set of possible asymptotic velocities or direction vectors for semi-infinite up-right paths is $\mathcal{U}=\{(t, 1-t): 0 \leqslant t \leqslant 1\}$, with relative interior $\operatorname{ri} \mathcal{U}=\{(t, 1-t): 0<t<1\}$.

We start with the results that are almost surely valid for all geodesics and directions.

Theorem 2.1. Assume (2.1). Then the following statements hold with $\mathbb{P}$-probability one.

(i) Each semi-infinite geodesic is $\mathbf{u}$-directed for some $\mathbf{u} \in \mathcal{U}$.

(ii) For $r \in\{1,2\}$ and each $x \in \mathbb{Z}^{2},\left\{x_{k}=x+k \mathbf{e}_{r}\right\}_{k \in \mathbb{Z}_{\geqslant 0}}$ is the only semi-infinite geodesic that satisfies $x_{0}=x$ and $\underline{\lim }_{k \rightarrow \infty} k^{-1} x_{k} \cdot \mathbf{e}_{3-r}=0$.

(iii) For each $\mathbf{u} \in \overline{\mathcal{U}}$ and $x \in \mathbb{Z}^{2}$ there exists a $\mathbf{u}$-directed semi-infinite geodesic that starts at $x$.

Parts (i)-(ii) together say that except for the trivial geodesics $x_{k}=x+k \mathbf{e}_{r}$ with constant increments, every semi-infinite geodesic is directed towards a vector $\mathbf{u}$ in the interior of the first quadrant.

The next theorem states properties that hold almost surely for a given direction $\mathbf{u}$. 
Theorem 2.2. Assume (2.1). Fix $\mathbf{u} \in$ riU. Then the following statements hold with $\mathbb{P}$-probability one.

(i) For each $x \in \mathbb{Z}^{2}$ there exists a unique $\mathbf{u}$-directed semi-infinite geodesic $\pi^{\mathbf{u}, x}=\left(\pi_{k}^{\mathbf{u}, x}\right)_{k \in \mathbb{Z} \geqslant 0}$ with initial point $\pi_{0}^{\mathbf{u}, x}=x$. Each point $\pi_{k}^{\mathbf{u}, x}$ is a Borel function of the weights $Y$. For each pair $x, y \in \mathbb{Z}^{2}$ these geodesics coalesce: that is, there exists $z \in \mathbb{Z}^{2}$ such that $\pi^{\mathbf{u}, x} \cap \pi^{\mathbf{u}, y}=\pi^{\mathbf{u}, z}$.

(ii) There is no bi-infinite geodesic in direction $\mathbf{u}$.

Let $\mathcal{T}_{\mathbf{u}}$ be the tree of all the $\mathbf{u}$-directed semi-infinite geodesics $\left\{\pi^{\mathbf{u}, x}: x \in \mathbb{Z}^{2}\right\}$. That is,

$$
\mathcal{T}_{\mathbf{u}}=\bigcup_{x \in \mathbb{Z}^{2}} \pi^{\mathbf{u} x}
$$

when we regard a geodesic as a collection of edges.

The dual lattice $\mathbb{Z}^{2 *}$ of $\mathbb{Z}^{2}$ is obtained by translating all the vertices and (nearest-neighbor) edges of $\mathbb{Z}^{2}$ by the vector $\mathbf{e}^{*}=\frac{1}{2}\left(\mathbf{e}_{1}+\mathbf{e}_{2}\right)=\left(\frac{1}{2}, \frac{1}{2}\right)$. An edge of $\mathbb{Z}^{2}$ and an edge of $\mathbb{Z}^{2 *}$ are dual if they cross each other or, equivalently, intersect at their midpoints. The unique dual of an edge $e$ of $\mathbb{Z}^{2}$ is denoted by $e^{*}$, and similarly $f^{*}$ denotes the dual of an edge $f$ of $\mathbb{Z}^{2 *}$. In particular, if $e=\left\{x-\mathbf{e}_{k}, x\right\}$ then $e^{*}=\left\{x-\mathbf{e}^{*}, x-\mathbf{e}^{*}+\mathbf{e}_{3-k}\right\}$, and $e^{* *}=e$.

The dual graph $\mathcal{T}_{\mathbf{u}}^{*}$ of the tree $\mathcal{T}_{\mathbf{u}}$ is defined through the edge duality:

$$
e^{*} \in \mathcal{T}_{\mathbf{u}}^{*} \quad \text { if and only if } e \notin \mathcal{T}_{\mathbf{u}} .
$$

Move the dual graph $\mathcal{T}_{\mathbf{u}}^{*}$ back on the original lattice by defining the graph

$$
\widetilde{\mathcal{T}}_{\mathbf{u}}=-\mathbf{e}^{*}-\mathcal{T}_{\mathbf{u}}^{*} \text {. }
$$

That is, edge $\left\{x-\mathbf{e}_{k}, x\right\} \in \widetilde{\mathcal{T}}_{\mathbf{u}}$ if and only if edge $\left\{-x-\mathbf{e}^{*},-x-\mathbf{e}^{*}+\mathbf{e}_{k}\right\} \in \mathcal{T}_{\mathbf{u}}^{*}$. The point of the next theorem is that $\widetilde{\mathcal{T}}_{\mathbf{u}}$ is also a tree of directed geodesics of an exponential CGM.

Theorem 2.3. Assume (2.1). Fix $\mathbf{u} \in \mathcal{U}$. Then there exists a collection $\tilde{Y}^{\mathbf{u}}=\left(\tilde{Y}_{x}^{\mathbf{u}}\right)_{x \in \mathbb{Z}^{2}}$ of i.i.d. $\operatorname{Exp}(1)$ weights on $(\Omega, \mathfrak{S}, \mathbb{P})$ with these properties.

(i) $\tilde{Y}^{\mathbf{u}}$ is a Borel function of the weights $Y$ in $(2.1)$ and $\tilde{Y}_{x}^{\mathbf{u}}\left(\theta_{y} \omega\right)=\tilde{Y}_{x-y}^{\mathbf{u}}(\omega) \forall x, y \in \mathbb{Z}^{2}$.

(ii) $\mathbb{P}$-almost surely $\widetilde{\mathcal{T}}_{\mathbf{u}}$ is the tree of the unique $\mathbf{u}$-directed semi-infinite geodesics of the LPP process $G^{Y^{\mathbf{u}}}$ defined as in (1.1) with $Y$ replaced by $\tilde{Y}^{\mathbf{u}}$.

In particular, the tree $\tilde{\mathcal{T}}_{\mathbf{u}}$ is equal in distribution to $\mathcal{T}_{\mathbf{u}}$. The dual graph $\mathcal{T}_{\mathbf{u}}^{*}$ is also $\mathbb{P}$-almost surely a tree.

The equality in distribution of $\mathcal{T}_{\mathbf{u}}$ and the (shifted and reflected) dual graph $\mathcal{T}_{\mathbf{u}}^{*}$ was originally proved by Pimentel (Lemma 2 in [17]). The weights $\tilde{Y}^{\mathbf{u}}$ are defined in (4.16) below.

As the final main results, we record some immediate consequences of the properties of Busemann functions, to be described in the next section. Distributional properties of the geodesic tree $\mathcal{T}_{\mathbf{u}}$ depend on a real parameter $\alpha \in(0,1)$ that is in bijective correspondence with the direction $\mathbf{u}=$ $\left(u_{1}, 1-u_{1}\right) \in \operatorname{ri} \mathcal{U}$. This bijection is defined by the equations

$$
\mathbf{u}=\mathbf{u}(\alpha)=\left(\frac{\alpha^{2}}{(1-\alpha)^{2}+\alpha^{2}}, \frac{(1-\alpha)^{2}}{(1-\alpha)^{2}+\alpha^{2}}\right) \Longleftrightarrow \alpha=\alpha(\mathbf{u})=\frac{\sqrt{u_{1}}}{\sqrt{u_{1}}+\sqrt{1-u_{1}}}
$$

For example, $\alpha$ gives the distribution of the first step of the geodesic:

$$
\mathbb{P}\left\{\pi_{1}^{\mathbf{u}, x}=x+\mathbf{e}_{1}\right\}=\alpha \quad \forall x \in \mathbb{Z}^{2} .
$$

This statement is proved after Lemma 4.1, after the proof of Theorem 2.1. Note however that the density of $\mathbf{e}_{1}$ steps along the $\mathbf{u}$-directed semi-infinite geodesic is $u_{1}$, which is different from $\alpha$, except in the special case $u_{1}=\alpha=\frac{1}{2}$. This points to the fact that understanding distributional properties along a geodesic is challenging. It is much easier to capture properties transversal to geodesics, as the next theorem illustrates. 


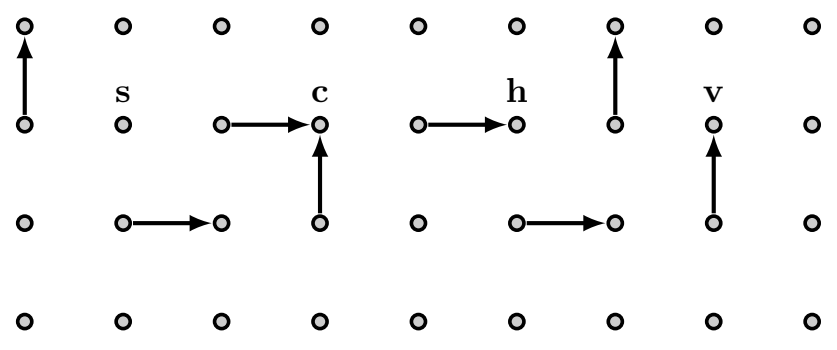

Figure 2.1. An example of a source (s), a coalescence point $(\mathbf{c})$, a horizontal point $(\mathbf{h})$, and a vertical point $(\mathbf{v})$. The arrows point from $x$ to $\pi_{1}^{\mathbf{u}, x}$. There is an arrow from each vertex $x$ but only the arrows needed for the definitions are displayed in the figure.

Call a point $z \in \mathbb{Z}^{2}$ a source if $z$ does not lie on $\pi^{\mathbf{u}, x}$ for any $x \neq z$. Call $z$ a coalescence point if there exist $x \neq y$ in $\mathbb{Z}^{2} \backslash\{z\}$ such that $\pi^{\mathbf{u}, z}=\pi^{\mathbf{u}, x} \cap \pi^{\mathbf{u}, y}$. Equivalently, $z$ is a source if $\pi_{1}^{\mathbf{u}, z-\mathbf{e}_{1}}=z-\mathbf{e}_{1}+\mathbf{e}_{2}$ and $\pi_{1}^{\mathbf{u}, z-\mathbf{e}_{2}}=z-\mathbf{e}_{2}+\mathbf{e}_{1}$, while $z$ is a coalescence point if $\pi_{1}^{\mathbf{u}, z-\mathbf{e}_{1}}=\pi_{1}^{\mathbf{u}, z-\mathbf{e}_{2}}=z$. To complete the list of possibilities, call $z$ a horizontal point if $\pi_{1}^{\mathbf{u}, z-\mathbf{e}_{1}}=z$ but $\pi_{1}^{\mathbf{u}, z-\mathbf{e}_{2}}=z-\mathbf{e}_{2}+\mathbf{e}_{1}$, and a vertical point if $\pi_{1}^{\mathbf{u}, z-\mathbf{e}_{1}}=z-\mathbf{e}_{1}+\mathbf{e}_{2}$ but $\pi_{1}^{\mathbf{u}, z-\mathbf{e}_{2}}=z$. See Figure 2.1 for an illustration.

Fix an antidiagonal $\mathcal{A}=\{(N+j,-j): j \in \mathbb{Z}\}$ of the lattice $\mathbb{Z}^{2}$, for some $N \in \mathbb{Z}$. Let $\xi_{j}$ be the random variable that takes one of the values $\{s, c, h, v\}$ to record whether point $(N+j,-j)$ is a source, a coalescence point, a horizontal point, or a vertical point.

Theorem 2.4. Assume (2.1). Fix $\mathbf{u} \in \mathcal{U}$ and let $\alpha=\alpha(\mathbf{u})$. Then $\left\{\xi_{j}\right\}_{j \in \mathbb{Z}}$ is a stationary Markov chain with state space $\{s, c, h, v\}$, transition matrix

$$
\mathbf{P}=\begin{gathered}
s \\
c \\
v \\
v
\end{gathered}\left[\begin{array}{cccc}
s & c & h & v \\
0 & 1-\alpha & \alpha & 0 \\
\alpha & 0 & 0 & 1-\alpha \\
0 & 1-\alpha & \alpha & 0 \\
\alpha & 0 & 0 & 1-\alpha
\end{array}\right]
$$

and invariant distribution

$$
\mu(s)=\mu(c)=\alpha(1-\alpha), \quad \mu(h)=\alpha^{2}, \quad \mu(v)=(1-\alpha)^{2} .
$$

In particular, both sources and coalescence points of semi-infinite geodesics in direction $\mathbf{u}=$ $\left(u_{1}, 1-u_{1}\right)$ have density

$$
\alpha(\mathbf{u})(1-\alpha(\mathbf{u}))=\frac{\sqrt{u_{1}\left(1-u_{1}\right)}}{\left(\sqrt{u_{1}}+\sqrt{1-u_{1}}\right)^{2}}
$$

on the lattice. This density is maximized at $1 / 4$ by the diagonal direction $\mathbf{u}=\left(\frac{1}{2}, \frac{1}{2}\right)$.

Organization of the rest of the paper. As mentioned, the purpose of the paper is to present a particular proof of Theorems 2.1-2.3. This proof has three main steps.

(i) Construction of the increment-stationary LPP process.

(ii) Proof of the existence and properties of Busemann functions, by using couplings with the increment-stationary LPP and monotonicity.

(iii) Control of geodesics with the Busemann functions.

Full details of steps (i) and (ii) are omitted from this paper because these steps are spelled out in lecture notes [19]. We review these arguments briefly in Section 3. The work of this paper goes towards step (iii). This is done in Section 4 that develops Busemann geodesics and proves the theorems of Section 2. A final Section 5 relates the geodesics constructed in Section 2 to competition interfaces. 


\section{INCREMENT-STATIONARY LPP AND BUSEMANN FUNCTIONS}

3.1. Preliminaries. A down-right path is a bi-infinite sequence $\mathcal{Y}=\left(y_{k}\right)_{k \in \mathbb{Z}}$ in $\mathbb{Z}^{2}$ such that $y_{k}-$ $y_{k-1} \in\left\{\mathbf{e}_{1},-\mathbf{e}_{2}\right\}$ for all $k \in \mathbb{Z}$. The lattice decomposes into a disjoint union $\mathbb{Z}^{2}=\mathcal{H}^{-} \cup \mathcal{Y} \cup \mathcal{H}^{+}$ where the two regions are

$$
\mathcal{H}^{-}=\left\{x \in \mathbb{Z}^{2}: \exists j \in \mathbb{Z}_{>0} \text { such that } x+j\left(\mathbf{e}_{1}+\mathbf{e}_{2}\right) \in \mathcal{Y}\right\}
$$

to the left of and below $\mathcal{Y}$ and

$$
\mathcal{H}^{+}=\left\{x \in \mathbb{Z}^{2}: \exists j \in \mathbb{Z}_{>0} \text { such that } x-j\left(\mathbf{e}_{1}+\mathbf{e}_{2}\right) \in \mathcal{Y}\right\}
$$

to the right of and above $\mathcal{Y}$.

It will be convenient to summarize certain properties of systems of exponential weights in the following definition.

Definition 3.1. Let $0<\alpha<1$. A stochastic process $\left\{\zeta_{x}, I_{x}, J_{x}, \eta_{x}: x \in \mathbb{Z}^{2}\right\}$ is an exponential- $\alpha$ last-passage percolation system if the following properties (a)-(b) hold.

(a) The process is stationary under lattice translations and has marginal distributions

$$
\zeta_{x}, \eta_{x} \sim \operatorname{Exp}(1), \quad I_{x} \sim \operatorname{Exp}(\alpha), \quad \text { and } \quad J_{x} \sim \operatorname{Exp}(1-\alpha) .
$$

For any down-right path $\mathcal{Y}=\left(y_{k}\right)_{k \in \mathbb{Z}}$ in $\mathbb{Z}^{2}$, the random variables

$$
\left\{\eta_{z}: z \in \mathcal{H}^{-}\right\}, \quad\left\{t\left(\left\{y_{k-1}, y_{k}\right\}\right): k \in \mathbb{Z}\right\}, \quad \text { and } \quad\left\{\zeta_{x}: x \in \mathcal{H}^{+}\right\}
$$

are all mutually independent, where the undirected edge variables $t(e)$ are defined as

$$
t(e)= \begin{cases}I_{x} & \text { if } e=\left\{x-\mathbf{e}_{1}, x\right\} \\ J_{x} & \text { if } e=\left\{x-\mathbf{e}_{2}, x\right\}\end{cases}
$$

(b) The following equations are in force at all $x \in \mathbb{Z}^{2}$ :

$$
\begin{aligned}
\eta_{x-\mathbf{e}_{1}-\mathbf{e}_{2}} & =I_{x-\mathbf{e}_{2}} \wedge J_{x-\mathbf{e}_{1}} \\
I_{x} & =\zeta_{x}+\left(I_{x-\mathbf{e}_{2}}-J_{x-\mathbf{e}_{1}}\right)^{+} \\
J_{x} & =\zeta_{x}+\left(I_{x-\mathbf{e}_{2}}-J_{x-\mathbf{e}_{1}}\right)^{-} .
\end{aligned}
$$

Equations (3.7)-(3.8) imply this counterpart of (3.6):

$$
\zeta_{x}=I_{x} \wedge J_{x}
$$

An exponential- $\alpha$ LPP system can be constructed explicitly in a quadrant as follows. Assume given independent weights $\left\{I_{i \mathbf{e}_{1}}: i \geqslant 1\right\}$ on the $x$-axis, $\left\{J_{j \mathbf{e}_{2}}: j \geqslant 1\right\}$ on the $y$-axis, and $\left\{\zeta_{x}: x \in \mathbb{Z}_{>0}^{2}\right\}$ in the bulk (interior) of the first quadrant, all with marginal distributions (3.3). Use equations (3.6)-(3.8) to define inductively in the northeast direction weights $\left\{\eta_{x-\mathbf{e}_{1}-\mathbf{e}_{2}}, I_{x}, J_{x}: x \in \mathbb{Z}_{>0}^{2}\right\}$. Then property

(a) from Definition 3.1 above can be verified inductively. Now $\left\{\zeta_{x+\mathbf{e}_{1}+\mathbf{e}_{2}}, I_{x+\mathbf{e}_{1}}, J_{x+\mathbf{e}_{2}}, \eta_{x}: x \in \mathbb{Z}_{\geqslant 0}^{2}\right\}$ is an exponential- $\alpha$ LPP system restricted to a quadrant.

Furthermore, if we define the LPP process $\left\{G_{x}^{\alpha}: x \in \mathbb{Z}_{\geqslant 0}^{2}\right\}$ by $G_{0}^{\alpha}=0$,

$$
G_{k \mathbf{e}_{1}}^{\alpha}=\sum_{i=1}^{k} I_{i \mathbf{e}_{1}} \text { for } k \geqslant 1, \quad G_{\ell \mathbf{e}_{2}}^{\alpha}=\sum_{j=1}^{\ell} J_{j \mathbf{e}_{2}} \text { for } \ell \geqslant 1,
$$

and inductively

$$
G_{x}^{\alpha}=\zeta_{x}+G_{x-\mathbf{e}_{1}}^{\alpha} \vee G_{x-\mathbf{e}_{2}}^{\alpha} \quad \text { for } x \in \mathbb{Z}_{>0}^{2},
$$


then $I$ and $J$ are the increments:

$$
I_{x}=G_{x}^{\alpha}-G_{x-\mathbf{e}_{1}}^{\alpha} \quad \text { and } \quad J_{x}=G_{x}^{\alpha}-G_{x-\mathbf{e}_{2}}^{\alpha} .
$$

All this is elementary to verify and contained in Theorem 3.1 of [19]. $\left\{G_{x}^{\alpha}: x \in \mathbb{Z}_{\geqslant 0}^{2}\right\}$ is an incrementstationary LPP process.

To produce an exponential- $\alpha$ LPP system on the full lattice as a function of the i.i.d. weights $Y$ of assumption (2.1), we take limits of LPP increments in the direction $\mathbf{u}(\alpha)$ determined by (2.5). For the statement we need a couple more definitions.

Define an order among direction vectors $\mathbf{u}=\left(u_{1}, 1-u_{1}\right)$ and $\mathbf{v}=\left(v_{1}, 1-v_{1}\right)$ in $\mathcal{U}$ according to the $\mathbf{e}_{1}$-coordinate:

$$
\mathbf{u}<\mathbf{v} \quad \text { if } \quad u_{1}<v_{1} .
$$

Geometrically: $\mathbf{u}<\mathbf{v}$ if $\mathbf{v}$ is below and to the right of $\mathbf{u}$. Bijection (2.5) preserves this order.

Definition 3.2. A measurable function $B: \Omega \times \mathbb{Z}^{2} \times \mathbb{Z}^{2} \rightarrow \mathbb{R}$ is a covariant cocycle if it satisfies these two conditions for $\mathbb{P}$-a.e. $\omega$ and all $x, y, z \in \mathbb{Z}^{2}$ :

$$
\begin{aligned}
B(\omega, x+z, y+z) & =B\left(\theta_{z} \omega, x, y\right) & & \text { (stationarity) } \\
B(\omega, x, y)+B(\omega, y, z) & =B(\omega, x, z) & & \text { (additivity). }
\end{aligned}
$$

$\mathcal{K}$ denotes the space of covariant cocycles $B$ such that $\mathbb{E}|B(x, y)|<\infty \forall x, y \in \mathbb{Z}^{2}$.

3.2. Busemann functions. Existence and properties of Busemann functions are summarized in the next theorem.

TheOrem 3.3. Assume (2.1). Then for each $\mathbf{u} \in$ riU there exist a covariant cocycle $B^{\mathbf{u}}=\left(B_{x, y}^{\mathbf{u}}\right)_{x, y \in \mathbb{Z}^{2}}$ and a family of random weights $X^{\mathbf{u}}=\left(X_{x}^{\mathbf{u}}\right)_{x \in \mathbb{Z}^{2}}$ on $(\Omega, \mathfrak{S}, \mathbb{P}, \Theta)$ with the following properties.

(i) For each $\mathbf{u} \in \mathrm{ri} \mathcal{U}$, process

$$
\left\{X_{x}^{\mathbf{u}}, B_{x-\mathbf{e}_{1}, x}^{\mathbf{u}}, B_{x-\mathbf{e}_{2}, x}^{\mathbf{u}}, Y_{x}: x \in \mathbb{Z}^{2}\right\}
$$

is an exponential- $\alpha(\mathbf{u})$ last-passage system as described in Definition 3.1. With $\mathbb{P}$-probability one, part (b) of Definition 3.1 holds simultaneously for all $\mathbf{u} \in \mathrm{ri} \mathcal{U}$.

(ii) There exists a single event $\Omega_{0}$ of full probability such that for all $\omega \in \Omega_{0}$, all $x \in \mathbb{Z}^{2}$ and all $\mathbf{u}<\mathbf{v}$ in ri $\mathcal{U}$ we have the inequalities

$$
B_{x, x+\mathbf{e}_{1}}^{\mathbf{u}}(\omega) \geqslant B_{x, x+\mathbf{e}_{1}}^{\mathbf{v}}(\omega) \quad \text { and } \quad B_{x, x+\mathbf{e}_{2}}^{\mathbf{u}}(\omega) \leqslant B_{x, x+\mathbf{e}_{2}}^{\mathbf{v}}(\omega) .
$$

Furthermore, for all $\omega \in \Omega_{0}$ and $x, y \in \mathbb{Z}^{2}$, the function $\mathbf{u} \mapsto B_{x, y}^{\mathbf{u}}(\omega)$ is right-continuous with left limits under the ordering (3.13).

(iii) For each fixed $\mathbf{v} \in$ ri $\mathcal{U}$ there exists an event $\Omega_{1}^{(\mathbf{v})}$ of full probability such that the following holds: for each $\omega \in \Omega_{1}^{(\mathbf{v})}$ and any sequence $v_{n} \in \mathbb{Z}^{2}$ such that $\left|v_{n}\right|_{1} \rightarrow \infty$ and

$$
\lim _{n \rightarrow \infty} \frac{v_{n}}{\left|v_{n}\right|_{1}}=\mathbf{v}
$$

we have the limits

$$
B_{x, y}^{\mathbf{v}}(\omega)=\lim _{n \rightarrow \infty}\left[G_{x, v_{n}}(\omega)-G_{y, v_{n}}(\omega)\right] \quad \forall x, y \in \mathbb{Z}^{2} .
$$

Furthermore, for all $\omega \in \Omega_{1}^{(\mathbf{v})}$ and $x, y \in \mathbb{Z}^{2}$,

$$
\lim _{\mathbf{u} \rightarrow \mathbf{v}} B_{x, y}^{\mathbf{u}}(\omega)=B_{x, y}^{\mathbf{v}}(\omega)
$$


Remark 3.4. The process $\mathbf{u} \mapsto B^{\mathbf{u}}$ is globally cadlag (part (ii)) and at each fixed $\mathbf{v}$ limit (3.17) holds almost surely. For each $x, y \in \mathbb{Z}^{2}, \mathbf{u} \mapsto B_{x, y}^{\mathbf{u}}$ is in fact a jump process [9]. The cadlag property is merely a convention. For certain purposes it can be useful to work with two processes $B_{+}^{\mathbf{u}}(x, y)$ and $B_{-}^{\mathbf{u}}(x, y)$ such that $\mathbf{u} \mapsto B_{+}^{\mathbf{u}}$ is right-continuous with left limits, $\mathbf{u} \mapsto B_{-}^{\mathbf{u}}$ is left-continuous with right limits, and $B_{+}^{\mathbf{u}}=B_{-}^{\mathbf{u}}$ almost surely for a given $\mathbf{u}$. Our results in Theorems 2.2-2.4 are almost sure statements for a fixed $\mathbf{u}$, and hence we could use either process $B_{+}^{\mathbf{u}}$ or $B_{-}^{\mathbf{u}}$.

Part (i) of Theorem 3.3 together with (3.6) and (3.9) imply

$$
Y_{x}=B_{x, x+\mathbf{e}_{1}}^{\mathbf{u}} \wedge B_{x, x+\mathbf{e}_{2}}^{\mathbf{u}}
$$

and

$$
X_{x}^{\mathbf{u}}=B_{x-\mathbf{e}_{1}, x}^{\mathbf{u}} \wedge B_{x-\mathbf{e}_{2}, x}^{\mathbf{u}} .
$$

From the exponential distributions of $B_{0, \mathbf{e}_{1}}^{\mathbf{u}}$ and $B_{0, \mathbf{e}_{2}}^{\mathbf{u}}$ and the explicit formula (1.3) of the shape function follows

$$
\left(\mathbb{E}\left[B_{0, \mathbf{e}_{1}}^{\mathbf{u}}\right], \mathbb{E}\left[B_{0, \mathbf{e}_{2}}^{\mathbf{u}}\right]\right)=\left(\frac{1}{\alpha(\mathbf{u})}, \frac{1}{1-\alpha(\mathbf{u})}\right)=\nabla g(\mathbf{u}) .
$$

This is natural since by (3.16) $B^{\mathbf{u}}$ can be viewed as the "microscopic gradient" of the passage time.

The next theorem gives strong uniqueness of the process $\left\{B^{\mathbf{u}}, X^{\mathbf{u}}\right\}$.

Theorem 3.5. Assume (2.1) and let $\left\{B^{\mathbf{u}}, X^{\mathbf{u}}: \mathbf{u} \in\right.$ ri $\left.\mathcal{U}\right\}$ be the process given by Theorem 3.3. Fix $0<\rho<1$. Suppose that on $(\Omega, \mathfrak{S}, \mathbb{P})$ there are random variables $\left(U_{x}, A_{x-\mathbf{e}_{1}, x}, A_{x-\mathbf{e}_{2}, x}\right)_{x \in \mathbb{Z}^{2}}$ such that $\left\{U_{x}, A_{x-\mathbf{e}_{1}, x}, A_{x-\mathbf{e}_{2}, x}, Y_{x}: x \in \mathbb{Z}^{2}\right\}$ is an exponential- $\rho$ last-passage system as described in Definition 3.1. Then $U_{x}=X_{x}^{\mathbf{u}(\rho)}, A_{x-\mathbf{e}_{1}, x}=B_{x-\mathbf{e}_{1}, x}^{\mathbf{u}(\rho)}$ and $A_{x-\mathbf{e}_{2}, x}=B_{x-\mathbf{e}_{2}, x}^{\mathbf{u}(\rho)}$ for all $x, \mathbb{P}$-almost surely.

3.3. The idea of the proof of Theorems 3.3 and 3.5. These theorems are proved in detail in Section 4 of lecture notes [19]. This type of proof was introduced first in the context of the positive-temperature log-gamma polymer in [12]. We sketch the main idea. The essential point for the message of this paper is that coalescence of geodesics is not used in the proof, only couplings, monotonicity, and properties of the increment-stationary LPP processes of (3.11).

In (3.16) let $\mathbf{v}=\mathbf{u}(\alpha)$ defined by (2.5). Construct an exponential- $\lambda$ LPP system in the quadrant $x+\mathbb{Z}_{\geqslant 0}^{2}$, as explained below (3.9). Use the i.i.d. $\operatorname{Exp}(1) \eta$-weights of this construction (defined by (3.6)) to define last-passage times $G_{x, y}$. Consider an $\mathbf{e}_{1}$-increment $G_{x, v_{n}}-G_{x+\mathbf{e}_{1}, v_{n}}$ in (3.16). Place the $I$ weights on the north and the $J$ weights on the east boundary of the rectangle $\left[x, v_{n}+\mathbf{e}_{1}+\mathbf{e}_{2}\right]$.

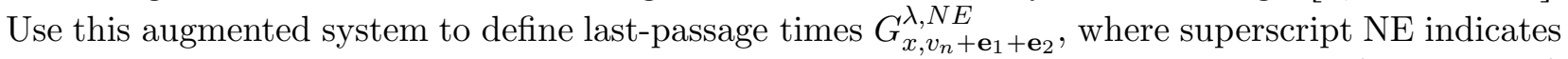
that the boundary weights are on the north and east. Then, by planar monotonicity (Lemma A.1) and by choosing $\lambda$ suitably, the upper bound

$$
G_{x, v_{n}}-G_{x+\mathbf{e}_{1}, v_{n}} \leqslant G_{x, v_{n}+\mathbf{e}_{1}+\mathbf{e}_{2}}^{\lambda, N E}-G_{x+\mathbf{e}_{1}, v_{n}+\mathbf{e}_{1}+\mathbf{e}_{2}}^{\lambda, N E}
$$

holds with high probability for large $n$. The right-hand increment above can be controlled because it comes from an increment-stationary LPP process. Similar reasoning yields a lower bound

$$
G_{x, v_{n}}-G_{x+\mathbf{e}_{1}, v_{n}} \geqslant G_{x, v_{n}+\mathbf{e}_{1}+\mathbf{e}_{2}}^{\rho, N E}-G_{x+\mathbf{e}_{1}, v_{n}+\mathbf{e}_{1}+\mathbf{e}_{2}}^{\rho, N E}
$$

with a different parameter $\rho$. After sending $v_{n}$ to infinity, the bounds are brought together by letting $\lambda$ and $\rho$ converge to $\alpha$.

This establishes the almost sure limit (3.16) for a countable dense set of directions v. Properties of the resulting processes $B^{\mathbf{v}}$ are derived from monotonicity and the increment-stationary LPP processes. The construction of the full process $\left\{B^{\mathbf{u}}: \mathbf{u} \in \operatorname{ri} \mathcal{U}\right\}$ is completed by taking right limits as $\mathbf{v} \searrow \mathbf{u}$ to get cadlag paths in the parameter $\mathbf{u}$. This proves Theorem 3.3. 
To prove the uniqueness in Theorem 3.5, the reasoning above is repeated: this time increment variables $A_{x-\mathbf{e}_{k}, x}$ are given, and planar monotonicity is used to sandwich them between Busemann limits from Theorem 3.3.

3.4. Midpoint problem. We quote one more result from [19] that is a corollary of the Busemann limits. We use this fact in the proof of Theorem 4.7 below to show the nonexistence of bi-infinite $B^{\mathbf{u}}$-geodesics. Let $\pi^{x, y}$ denote the (almost surely unique) geodesic for $G_{x, y}$ defined by (1.1).

TheOREM 3.6. Assume (2.1) and fix $\mathbf{u} \in$ riU. Let $u_{n} \leqslant z_{n} \leqslant v_{n}$ be three sequences on $\mathbb{Z}^{2}$ that satisfy the following conditions: $u_{n}$ and $v_{n}$ can be random but $z_{n}$ is not (that is, $u_{n}$ and $v_{n}$ can be measurable functions of $\omega$ but $z_{n}$ does not depend on $\left.\omega\right),\left|v_{n}-z_{n}\right|_{1} \rightarrow \infty,\left|z_{n}-u_{n}\right|_{1} \rightarrow \infty$, and

$$
\lim _{n \rightarrow \infty} \frac{v_{n}-z_{n}}{\left|v_{n}-z_{n}\right|_{1}}=\lim _{n \rightarrow \infty} \frac{z_{n}-u_{n}}{\left|z_{n}-u_{n}\right|_{1}}=\mathbf{u} \text {. }
$$

Then $\lim _{n \rightarrow \infty} \mathbb{P}\left\{z_{n} \in \pi^{u_{n}, v_{n}}\right\}=0$.

This theorem is proved for deterministic $u_{n}, v_{n}$ in lecture notes [19] as Theorem 4.12 on p. 174 . The same argument proves the version above for random $u_{n}, v_{n}$ and appears in the arXiv version of [19]. The proof proceeds by expressing the condition $z_{n} \in \pi^{u_{n}, v_{n}}$ in terms of increments of $G_{x, y}$ and then taking the Busemann limits (3.16).

\section{Busemann geodesics And PROOFs of the MAin theorems}

4.1. Busemann geodesics. Let $\left\{B^{\mathbf{u}}: \mathbf{u} \in \mathrm{ri} \mathcal{U}\right\}$ be the covariant integrable cocycles constructed in Theorem 3.3. We write interchangeably $B^{\mathbf{u}}(x, y, \omega)=B_{x, y}^{\mathbf{u}}(\omega)$. For each direction $\mathbf{u} \in$ ri $\mathcal{U}$ and initial point $x \in \mathbb{Z}^{2}$ construct a semi-infinite random up-right lattice path $\mathbf{b}^{\mathbf{u}, x}(\omega)=\left\{\mathbf{b}_{k}^{\mathbf{u}, x}(\omega)\right\}_{k \in \mathbb{Z} \geqslant 0}$ by following minimal increments of $B^{\mathbf{u}}$ :

$$
\begin{aligned}
& \mathbf{b}_{0}^{\mathbf{u}, x}(\omega)=x, \quad \text { and for } k \geqslant 0 \\
& \mathbf{b}_{k+1}^{\mathbf{u}, x}(\omega)= \begin{cases}\mathbf{b}_{k}^{\mathbf{u}, x}(\omega)+\mathbf{e}_{1}, & \text { if } B_{\mathbf{b}_{k}^{\mathbf{u}, x}, \mathbf{b}_{k}^{\mathbf{u}, x}+\mathbf{e}_{1}}^{\mathbf{u}}(\omega) \leqslant B_{\mathbf{b}_{k}^{\mathbf{u}, x}, \mathbf{b}_{k}^{\mathbf{u}, x}+\mathbf{e}_{2}}^{\mathbf{u}}(\omega) \\
\mathbf{b}_{k}^{\mathbf{u}, x}(\omega)+\mathbf{e}_{2}, & \text { if } B_{\mathbf{b}_{k}^{\mathbf{u}, x}, \mathbf{b}_{k}^{\mathbf{u}, x}+\mathbf{e}_{2}}^{\mathbf{u}}(\omega)<B_{\mathbf{b}_{k}^{\mathbf{u}, x}, \mathbf{b}_{k}^{\mathbf{u}, x}+\mathbf{e}_{1}}^{\mathbf{u}}(\omega) .\end{cases}
\end{aligned}
$$

The tie-breaking rule in favor of $\mathbf{e}_{1}$ is a convention we follow henceforth. For a given $\mathbf{u}$ the case of equality on the right-hand side of the two-case formula happens with probability zero because $B_{x, x+\mathbf{e}_{1}}^{\mathbf{u}}$ and $B_{x, x+\mathbf{e}_{2}}^{\mathbf{u}}$ are independent exponential random variables. Pictorially, to each point $z$ attach an arrow that points from $z$ to $\mathbf{b}_{1}^{\mathbf{u}, z}$. The path $\mathbf{b}^{\mathbf{u}, x}$ is constructed by starting at $x$ and following the arrows. By (3.18),

$$
Y_{\mathbf{b}_{k}^{\mathbf{u}, x}}=B^{\mathbf{u}}\left(\mathbf{b}_{k}^{\mathbf{u}, x}, \mathbf{b}_{k+1}^{\mathbf{u}, x}\right) \quad \text { for } k \geqslant 0 .
$$

We shall call $\mathbf{b}^{\mathbf{u}, x}$ the $B^{\mathbf{u}}$-geodesic from $x$. This term is justified by the next lemma. Since the processes $B^{\mathbf{u}}$ arise as Busemann functions, we can also call these geodesics Busemann geodesics.

LEMMA 4.1.

(i) $\mathbf{b}^{\mathbf{u}, x}$ is a semi-infinite geodesic for the LPP process (1.1). For all $0 \leqslant m<n$,

$$
G\left(\mathbf{b}_{m}^{\mathbf{u}, x}, \mathbf{b}_{n}^{\mathbf{u}, x}\right)=B^{\mathbf{u}}\left(\mathbf{b}_{m}^{\mathbf{u}, x}, \mathbf{b}_{n}^{\mathbf{u}, x}\right)+Y_{\mathbf{b}_{n}^{\mathbf{u}, x}} .
$$

(ii) There exists an event $\Omega_{2}$ such that $\mathbb{P}\left(\Omega_{2}\right)=1$ and for all $\omega \in \Omega_{2}$ the following properties hold $\forall \mathbf{u}, \mathbf{v} \in \operatorname{ri} \mathcal{U}$. If $\mathbf{u}<\mathbf{v}$, then $\mathbf{b}^{\mathbf{v}, x}$ stays always (weakly) to the right and below $\mathbf{b}^{\mathbf{u}, x}$. Furthermore, geodesic $\mathbf{b}^{\mathbf{u}, x}$ is $\mathbf{u}$-directed:

$$
\lim _{n \rightarrow \infty} \frac{\mathbf{b}_{n}^{\mathbf{u}, x}}{n}=\mathbf{u} \quad \forall x \in \mathbb{Z}^{2}
$$


(iii) For each fixed $\mathbf{v} \in$ riU there exists an event $\Omega_{3}^{(\mathbf{v})}$ such that $\mathbb{P}\left(\Omega_{3}^{(\mathbf{v})}\right)=1$ and the following properties hold for each $\omega \in \Omega_{3}^{(\mathbf{v})}$ and $x \in \mathbb{Z}^{2}: \forall k \in \mathbb{Z}_{\geqslant 0}, \mathbf{b}_{k}^{\mathbf{u}, x} \rightarrow \mathbf{b}_{k}^{\mathbf{v}, x}$ as $\mathbf{u} \rightarrow \mathbf{v}$ in ri $\mathcal{U}$, and furthermore, $\mathbf{b}^{\mathbf{v}, x}$ is the unique semi-infinite $\mathbf{v}$-directed geodesic out of $x$. In particular, the geodesic tree $\mathcal{T}_{\mathbf{v}}$ defined by (2.2) can be expressed as

$$
\mathcal{T}_{\mathbf{v}}=\bigcup_{x \in \mathbb{Z}^{2}} \mathbf{b}^{\mathbf{v}, x}
$$

where again geodesics are regarded as collections of edges.

Proof. Part (i). Let $x_{0, n}$ be any path from $x_{0}=\mathbf{b}_{0}^{\mathbf{u}, x}=x$ to $x_{n}=\mathbf{b}_{n}^{\mathbf{u}, x}$. By (3.18) and (4.2),

$$
\begin{aligned}
\sum_{k=0}^{n} Y_{x_{k}} & \leqslant \sum_{k=0}^{n-1} B^{\mathbf{u}}\left(x_{k}, x_{k+1}\right)+Y_{x_{n}}=B^{\mathbf{u}}\left(x_{0}, x_{n}\right)+Y_{x_{n}}=B^{\mathbf{u}}\left(\mathbf{b}_{0}^{\mathbf{u}, x}, \mathbf{b}_{n}^{\mathbf{u}, x}\right)+Y_{\mathbf{b}_{n}^{\mathbf{u}, x}} \\
& =\sum_{k=0}^{n-1} B^{\mathbf{u}}\left(\mathbf{b}_{k}^{\mathbf{u}, x}, \mathbf{b}_{k+1}^{\mathbf{u}, x}\right)+Y_{\mathbf{b}_{n}^{\mathbf{u}, x}}=\sum_{k=0}^{n} Y_{\mathbf{b}_{k}^{\mathbf{u}, x} .}
\end{aligned}
$$

Thus for any $n$, the segment $\mathbf{b}_{0, n}^{\mathbf{u}, x}$ is a geodesic between its endpoints.

Part (ii). The ordering of Busemann geodesics follows from the monotonicity (3.14) of the Busemann functions.

For the limit (4.4) consider first fixed $\mathbf{u} \in \mathrm{ri \mathcal {U }}$. Recall the mean vector of $B^{\mathbf{u}}$ from (3.20). The cocycle ergodic theorem (Theorem B.1 in the Appendix) applies to the mean-zero cocycle

$$
F(\omega, x, y)=-B^{\mathbf{u}}(x, y, \omega)+\nabla g(\mathbf{u}) \cdot(y-x)
$$

by virtue of the bound $F\left(\omega, 0, \mathbf{e}_{i}\right) \leqslant-Y_{0}+C$ that comes from (3.18). By translation-invariance, if (4.4) is proved for $x=0$ it follows for all $x$. Since $\mathbf{b}^{\mathbf{u}, 0} \subset \mathbb{Z}_{\geqslant 0}^{2}, g\left(\mathbf{b}_{n}^{\mathbf{u}, 0}\right)$ is defined for the shape function $g$ in (1.3). Then by the homogeneity of $g,(4.3),(1.4)$, and Theorem B.1,

$$
\begin{aligned}
& g\left(\frac{\mathbf{b}_{n}^{\mathbf{u}, 0}}{n}\right)-\nabla g(\mathbf{u}) \cdot \frac{\mathbf{b}_{n}^{\mathbf{u}, 0}}{n} \\
& \quad=\frac{1}{n}\left[g\left(\mathbf{b}_{n}^{\mathbf{u}, 0}\right)-G\left(0, \mathbf{b}_{n}^{\mathbf{u}, 0}\right)\right]+\frac{1}{n}\left[B^{\mathbf{u}}\left(0, \mathbf{b}_{n}^{\mathbf{u}, 0}\right)-\nabla g(\mathbf{u}) \cdot \mathbf{b}_{n}^{\mathbf{u}, 0}\right]+\frac{Y_{\mathbf{b}_{n}^{\mathbf{u}, 0}}}{n} \\
& \quad \longrightarrow \quad \text { almost surely as } n \rightarrow \infty .
\end{aligned}
$$

All the limit points of $\mathbf{b}_{n}^{\mathbf{u}, 0} / n$ lie on $\mathcal{U}$. As a differentiable, concave and homogeneous function, $g$ satisfies $g(\xi)=\nabla g(\xi) \cdot \xi$ for all $\xi \in \mathbb{R}_{>0}^{2}$. Since $g$ is strictly concave on $\mathcal{U}$, for every $\delta>0$ there exists $\varepsilon>0$ such that

$$
g(\mathbf{v}) \leqslant \nabla g(\mathbf{u}) \cdot \mathbf{v}-\varepsilon \quad \text { for } \mathbf{v} \in \mathcal{U} \text { such that }|\mathbf{v}-\mathbf{u}| \geqslant \delta .
$$

Thus the limit above forces $\mathbf{b}_{n}^{\mathbf{u}, 0} / n \rightarrow \mathbf{u}$ almost surely.

Let $\Omega_{2}$ be the event on which limit (4.4) happens for a countable dense set of directions $\mathbf{u} \in$ ri $\mathcal{U}$ and all $x \in \mathbb{Z}^{2}$. The limit extends simultaneously to all $\mathbf{u} \in$ ri $\mathcal{U}$ on the event $\Omega_{2}$ by virtue of the ordering of the geodesics $\mathbf{b}^{\mathbf{u}, x}$.

Part (iii). Let $\Omega_{3}^{(\mathbf{v})}$ be the event on which limits (3.17) hold, uniqueness of finite geodesics holds, equality on the right-hand side of (4.1) does not happen for the fixed $\mathbf{v}$, and part (ii) above holds. On this event $\mathbf{b}_{k}^{\mathbf{u}, x} \rightarrow \mathbf{b}_{k}^{\mathbf{v}, x}$ as $\mathbf{u} \rightarrow \mathbf{v}$ because, inductively in $k$, (4.1) chooses the same step for all $\mathbf{u}$ close enough to $\mathbf{v}$ by virtue of (3.17).

Let $\pi=\left(\pi_{i}\right)_{i \in \mathbb{Z}_{\geqslant 0}}$ be a $\mathbf{v}$-directed semi-infinite geodesic from $\pi_{0}=x$. Let $\mathbf{u}<\mathbf{v}<\mathbf{w}$ in ri $\mathcal{U}$. By the directedness (4.4), after some (random but finite) number of steps $\pi$ remains strictly between $\mathbf{b}^{\mathbf{u}, x}$ and $\mathbf{b}^{\mathbf{w}, x}$. Then it follows that $\pi$ remains for all time weakly between $\mathbf{b}^{\mathbf{u}, x}$ and $\mathbf{b}^{\mathbf{w}, x}$. For if $\pi$ 
ever went strictly to the left of $\mathbf{b}^{\mathbf{u}, x}$, it would have to eventually intersect $\mathbf{b}^{\mathbf{u}, x}$ at some later point $\pi_{m}=\mathbf{b}_{m}^{\mathbf{u}, x}$. Then there would be two distinct geodesics $\pi_{0, m}$ and $\mathbf{b}_{0, m}^{\mathbf{u}, x}$ from $x$ to $\pi_{m}$, in violation of the uniqueness of finite geodesics. Similarly $\pi$ cannot go strictly to the right of $\mathbf{b}^{\mathbf{w}, x}$.

Letting $\mathbf{u} \rightarrow \mathbf{v}$ and $\mathbf{w} \rightarrow \mathbf{v}$ shows that $\pi$ must coincide with $\mathbf{b}^{\mathbf{v}, x}$.

Proof of Theorem 2.1. Part (i). Let $\Omega_{4}$ be the full probability event on which finite geodesics are unique and limits (4.4) hold for all $x \in \mathbb{Z}^{2}$ and all $\mathbf{u} \in$ ri $\mathcal{U}$. Fix $\omega \in \Omega_{4}$. Let $x_{\bullet}=\left(x_{n}\right)_{n \geqslant n_{0}}$ be a semi-infinite geodesic at this sample point $\omega$. We can assume it indexed so that $x_{n} \cdot\left(\mathbf{e}_{1}+\mathbf{e}_{2}\right)=n$. Suppose

$$
\underline{u}_{1}=\underline{\lim } \frac{x_{n} \cdot \mathbf{e}_{1}}{n}<\varlimsup_{n \rightarrow \infty} \frac{x_{n} \cdot \mathbf{e}_{1}}{n}=\bar{u}_{1} .
$$

Then necessarily $0 \leqslant \underline{u}_{1}<\bar{u}_{1} \leqslant 1$. Pick a vector $\mathbf{u} \in \operatorname{ri} \mathcal{U}$ between $\underline{\mathbf{u}}=\left(\underline{u}_{1}, 1-\underline{u}_{1}\right)$ and $\overline{\mathbf{u}}=\left(\bar{u}_{1}, 1-\bar{u}_{1}\right)$. Then infinitely often $x$. is strictly to the left of, strictly to the right of, and crosses $\mathbf{b}^{\mathbf{u}, x_{n_{0}}}$. This violates the uniqueness of finite geodesics. Consequently (4.7) cannot happen on $\Omega_{4}$ and hence all semi-infinite geodesics have a direction.

Part (ii). We prove the case $\mathbf{e}_{1}$ for $x=0$. Fix a sequence $\mathbf{w}_{1}<\mathbf{w}_{2}<\cdots<\mathbf{w}_{k}<\cdots$ in ri $\mathcal{U}$ such that $\mathbf{w}_{k} \rightarrow \mathbf{e}_{1}$. By Theorem 3.3, $B_{0, \mathbf{e}_{2}}^{\mathbf{w}_{k}} \sim \operatorname{Exp}\left(1-\alpha\left(\mathbf{w}_{k}\right)\right)$. Since $1-\alpha\left(\mathbf{w}_{k}\right) \rightarrow 0$,

$$
B_{0, \mathbf{e}_{2}}^{\mathbf{w}_{k}} \rightarrow \infty \quad \text { almost surely as } k \rightarrow \infty
$$

by the monotonicity (3.14). While retaining $\mathbb{P}\left(\Omega_{4}\right)=1$, modify the event $\Omega_{4}$ so that (4.8) holds on $\Omega_{4}$, and further intersect it with the (countably many full probability) events $\Omega_{1}^{\left(\mathbf{w}_{k}\right)}$ from Theorem 3.3(iii). Now the Busemann limit (3.16) holds on $\Omega_{4}$ for $\mathbf{v}=\mathbf{w}_{k}$ for each $k$.

Fix $\omega \in \Omega_{4}$. Suppose that at this $\omega$ there is a semi-infinite geodesic $\pi=\left\{\pi_{n}\right\}_{n \in \mathbb{Z} \geqslant 0}$ such that $\pi_{0}=0, \pi_{\ell}=(\ell-1,1)$ for some $\ell \geqslant 1$, and $\underline{\lim }_{n \rightarrow \infty} n^{-1} \pi_{n} \cdot \mathbf{e}_{2}=0$. We derive a contradiction from this.

By connecting $\mathbf{e}_{2}=(0,1)$ to the point $\pi_{\ell}=(\ell-1,1)$ (now fixed for the present) with a horizontal path, we get the lower bound

$$
G_{\mathbf{e}_{2}, \pi_{n}} \geqslant \sum_{i=0}^{\ell-1} \omega_{(i, 1)}+G_{\pi_{\ell+1}, \pi_{n}} \quad \text { for } n>\ell .
$$

That $\pi$ is a geodesic from $\pi_{0}=0$ implies $G_{0, \pi_{n}}=G_{0, \pi_{\ell}}+G_{\pi_{\ell+1}, \pi_{n}}$ for $n>\ell$. Thus

$$
G_{0, \pi_{n}}-G_{\mathbf{e}_{2}, \pi_{n}} \leqslant G_{0, \pi_{\ell}}-\sum_{i=0}^{\ell-1} \omega_{(i, 1)} \quad \text { for all } n>\ell .
$$

For each $k$, fix a sequence $\left\{w_{n, k}\right\}_{n \geqslant 0}$ in $\mathbb{Z}_{\geqslant 0}^{2}$ such that $\left|w_{n, k}\right|_{1}=n$ and $\lim _{n \rightarrow \infty} n^{-1} w_{n, k}=\mathbf{w}_{k}$. By the assumptions $\underline{\lim } n^{-1} \pi_{n} \cdot \mathbf{e}_{2}=0$ and $\mathbf{w}_{k} \in \mathrm{ri} \mathcal{U}$, and by Lemma A.1, there are infinitely many indices $n$ such that

$$
G_{0, \pi_{n}}-G_{\mathbf{e}_{2}, \pi_{n}} \geqslant G_{0, w_{n, k}}-G_{\mathbf{e}_{2}, w_{n, k}} .
$$

Hence by the Busemann limit (3.16),

$$
\varlimsup_{n \rightarrow \infty}\left[G_{0, \pi_{n}}-G_{\mathbf{e}_{2}, \pi_{n}}\right] \geqslant B_{0, \mathbf{e}_{2}}^{\mathbf{w}_{k}} .
$$

Limit (4.8) now contradicts (4.9) because the right-hand side of (4.9) is fixed and finite.

Part (iii). The family $\left\{\mathbf{b}^{\mathbf{u}, x}: \mathbf{u} \in \operatorname{ri} \mathcal{U}, x \in \mathbb{Z}^{2}\right\}$ gives a $\mathbf{u}$-directed semi-infinite geodesic for each $\mathbf{u} \in$ ri $\mathcal{U}$ and each starting point $x$. A semi-infinite geodesic in direction $\mathbf{e}_{r}$ from $x$ is defined trivially by $x_{k}=x+k \mathbf{e}_{r}$ for $k \geqslant 0$. 
Proof of (2.6). By part (iii) of Lemma 4.1 and by (4.1),

$$
\mathbb{P}\left\{\pi_{1}^{\mathbf{u}, x}=x+\mathbf{e}_{1}\right\}=\mathbb{P}\left\{\mathbf{b}_{1}^{\mathbf{u}, x}=x+\mathbf{e}_{1}\right\}=\mathbb{P}\left\{B_{x, x+\mathbf{e}_{1}}^{\mathbf{u}} \leqslant B_{x, x+\mathbf{e}_{2}}^{\mathbf{u}}\right\}=\alpha .
$$

The last equality is due to the fact that $B_{x, x+\mathbf{e}_{1}}^{\mathbf{u}}$ and $B_{x, x+\mathbf{e}_{2}}^{\mathbf{u}}$ are independent exponential random variables with rates $\alpha$ and $1-\alpha$, respectively. This comes from part (i) of Theorem 3.3 because $\left(x+\mathbf{e}_{2}, x, x+\mathbf{e}_{1}\right)$ is a segment of a down-right path.

Remark 4.2. If two separate Busemann processes $B_{+}^{\mathbf{u}}$ and $B_{-}^{\mathbf{u}}$ are constructed as indicated in Remark 3.4, then two Busemann geodesics $\mathbf{b}^{\mathbf{u}, x,+}$ and $\mathbf{b}^{\mathbf{u}, x,-}$ would be constructed by (4.1). Lemma 4.1 would hold for both families. Furthermore, $\mathbf{b}^{\mathbf{u}, x,+}$ would always stay weakly to the right and below $\mathbf{b}^{\mathbf{u}, x,-}$.

In view of Lemma 4.1(iii), to complete the proof of Theorem 2.2 , it suffices to prove that, $\mathbb{P}$ almost surely for a fixed $\mathbf{u} \in \operatorname{ri} \mathcal{U}$, geodesics $\mathbf{b}^{\mathbf{u}, x}$ and $\mathbf{b}^{\mathbf{u}, y}$ coalesce and that there is no bi-infinite

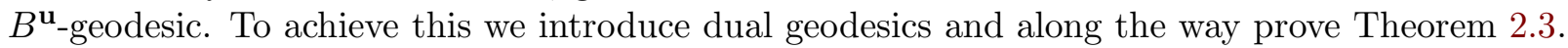

4.2. South-west and dual geodesics. Define south-west $B^{\mathbf{u}}$-geodesics $\mathbf{b}^{\mathrm{sw}, \mathbf{u}, x}(\omega)$ by following minimal south-west increments of $B^{\mathbf{u}}$ :

$$
\begin{aligned}
& \mathbf{b}_{0}^{\mathrm{sw}, \mathbf{u}, x}(\omega)=x, \quad \text { and for } k \geqslant 0
\end{aligned}
$$

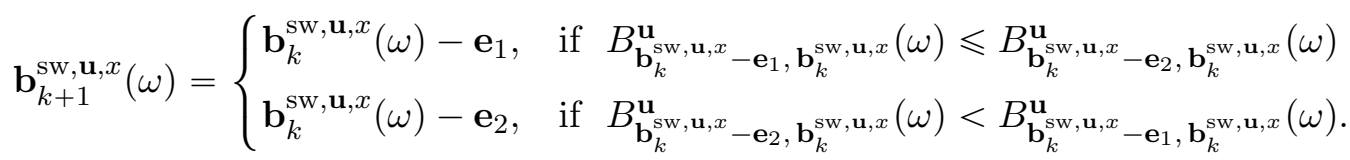

By (3.19),

$$
X_{\mathbf{b}_{k}^{\mathrm{sw}, \mathbf{u}, x}}^{\mathbf{u}}=B^{\mathbf{u}}\left(\mathbf{b}_{k+1}^{\mathrm{sw}, \mathbf{u}, x}, \mathbf{b}_{k}^{\mathrm{sw}, \mathbf{u}, x}\right) \text { for } k \geqslant 0 .
$$

Define an LPP process in terms of the weights $X^{\mathbf{u}}$ :

$$
G_{x, y}^{X^{\mathbf{u}}}=G^{X^{\mathbf{u}}}(x, y)=\max _{x, \in \Pi_{x, y}} \sum_{k=0}^{|y-x|_{1}} X_{x_{k}}^{\mathbf{u}} \quad \text { for } x \leqslant y \text { on } \mathbb{Z}^{2} .
$$

We think of this LPP process as pointing down and left, but do not alter the ordering $x \leqslant y$ in the notation $G_{x, y}^{X^{\mathbf{u}}}$.

Lemma 4.3. Fix $\mathbf{u} \in \operatorname{ri\mathcal {U}}$.

(i) $\mathbf{b}^{\mathrm{sw}, \mathbf{u}, x}$ is a semi-infinite down-left geodesic for LPP process $G^{X^{\mathbf{u}}}$ defined by (4.12). For all $0 \leqslant m<n$,

$$
G^{X^{\mathbf{u}}}\left(\mathbf{b}_{n}^{\mathrm{sw}, \mathbf{u}, x}, \mathbf{b}_{m}^{\mathrm{sw}, \mathbf{u}, x}\right)=B^{\mathbf{u}}\left(\mathbf{b}_{n}^{\mathrm{sw}, \mathbf{u}, x}, \mathbf{b}_{m}^{\mathrm{sw}, \mathbf{u}, x}\right)+X_{\mathbf{b}_{n}^{\mathrm{sw}, \mathbf{u}, x}}^{\mathbf{u}} .
$$

(ii) We have the $\mathbb{P}$-almost sure direction

$$
\lim _{n \rightarrow \infty} \frac{\mathbf{b}_{n}^{\mathrm{sw}, \mathbf{u}, x}}{n}=-\mathbf{u} \quad \forall x \in \mathbb{Z}^{2} .
$$

(iii) $B^{\mathbf{u}}$ is the Busemann function for LPP process $G^{X^{\mathbf{u}}}$ in direction - $\mathbf{u}$. Precisely, on the event $\Omega_{1}^{(\mathbf{u})}$ of Theorem 3.3(iii) and for any sequence $v_{n} \in \mathbb{Z}^{2}$ such that $\left|v_{n}\right|_{1} \rightarrow \infty$ and $v_{n} /\left|v_{n}\right|_{1} \rightarrow-\mathbf{u}$,

$$
B_{x, y}^{\mathbf{u}}=\lim _{n \rightarrow \infty}\left[G_{v_{n}, y}^{X^{\mathbf{u}}}-G_{v_{n}, x}^{X^{\mathbf{u}}}\right] \quad \forall x, y \in \mathbb{Z}^{2} .
$$

Proof. Part (i) is proved as in Lemma 4.1, by utilizing (3.19) and (4.11).

Define a process $\left(\widetilde{X}, \widetilde{B}^{\mathbf{u}}, \tilde{Y}^{\mathbf{u}}\right)$ by setting

$$
\tilde{X}_{x}=Y_{-x}, \widetilde{B}_{x, y}^{\mathbf{u}}=B_{-y,-x}^{\mathbf{u}}, \text { and } \tilde{Y}_{x}^{\mathbf{u}}=X_{-x}^{\mathbf{u}} \quad \forall x, y \in \mathbb{Z}^{2} .
$$


Properties of $\left(X^{\mathbf{u}}, B^{\mathbf{u}}, Y\right)$ given in Theorem 3.3 imply that $\left\{\widetilde{X}_{x}, \widetilde{B}_{x-\mathbf{e}_{1}, x}^{\mathbf{u}}, \widetilde{B}_{x-\mathbf{e}_{2}, x}^{\mathbf{u}}, \widetilde{Y}_{x}^{\mathbf{u}}\right\}_{x \in \mathbb{Z}^{2}}$ is an exponential- $\alpha(\mathbf{u})$ LPP system. By Theorem $3.5, \widetilde{B}^{\mathbf{u}}$ is the Busemann function in direction $\mathbf{u}$ of the LPP process $G^{\widetilde{Y}^{\mathbf{u}}}$ defined by (1.1) but with weights $\tilde{Y}^{\mathbf{u}}$.

For part (ii), apply definition (4.1) to $\widetilde{B}^{\mathbf{u}}$ and compare the outcome with (4.10) to conclude that $-\mathbf{b}^{\mathrm{sw}, \mathbf{u}, x}$ is the $\widetilde{B}^{\mathbf{u}}$ geodesic that starts at $-x$. Limit (4.4) applied to the LPP process $G^{\widetilde{Y}^{\mathbf{u}}}$ gives (4.14).

Part (iii) follows from

$$
\lim _{n \rightarrow \infty}\left[G_{v_{n}, y}^{X^{\mathbf{u}}}-G_{v_{n}, x}^{X^{\mathbf{u}}}\right]=\lim _{n \rightarrow \infty}\left[G_{-y,-v_{n}}^{\tilde{Y}^{\mathbf{u}}}-G_{-x,-v_{n}}^{\tilde{Y}^{\mathbf{u}}}\right]=\widetilde{B}_{-y,-x}^{\mathbf{u}}=B_{x, y}^{\mathbf{u}} .
$$

Define dual $B^{\mathbf{u}}$-geodesics $\mathbf{b}^{*, \mathbf{u}, z}$ on the dual lattice $\mathbb{Z}^{2 *}$ by shifting south-west geodesics by $\mathbf{e}^{*}=$ $\left(\frac{1}{2}, \frac{1}{2}\right)$ :

$$
\mathbf{b}_{k}^{*, \mathbf{u}, z}=\mathbf{b}_{k}^{\mathrm{sw}, \mathbf{u}, z+\mathbf{e}^{*}}-\mathbf{e}^{*} \quad \text { for } z \in \mathbb{Z}^{2 *} \text { and } k \geqslant 0 .
$$

Lemma 4.4. Fix $\mathbf{u} \in \mathrm{ri \mathcal {U }}$. Then an edge e lies on some geodesic $\mathbf{b}^{\mathbf{u}, x}$ if and only if its dual edge $e^{*}$ does not lie on any dual geodesic $\mathbf{b}^{*, \mathbf{u}, z}$. In particular, the family $\left\{\mathbf{b}^{\mathbf{u}, x}: x \in \mathbb{Z}^{2}\right\}$ of $B^{\mathbf{u}}$-geodesics and the family $\left\{\mathbf{b}^{*, \mathbf{u}, z}: z \in \mathbb{Z}^{2 *}\right\}$ of dual $B^{\mathbf{u}}$-geodesics never cross each other.

Proof. We need to check that, for $x \in \mathbb{Z}^{2}, \mathbf{b}_{1}^{\mathbf{u}, x}=x+\mathbf{e}_{1}$ if and only if $\mathbf{b}_{1}^{*, \mathbf{u}, x+\mathbf{e}^{*}}=x+\mathbf{e}^{*}-\mathbf{e}_{1}$.

$$
\begin{aligned}
& \mathbf{b}_{1}^{*, \mathbf{u}, x+\mathbf{e}^{*}}=x+\mathbf{e}^{*}-\mathbf{e}_{1} \Longleftrightarrow \mathbf{b}_{1}^{\mathrm{sw}, \mathbf{u}, x+\mathbf{e}_{1}+\mathbf{e}_{2}}=x+\mathbf{e}_{2} \\
& \Longleftrightarrow B_{x+\mathbf{e}_{2}, x+\mathbf{e}_{1}+\mathbf{e}_{2}}^{\mathbf{u}} \leqslant B_{x+\mathbf{e}_{1}, x+\mathbf{e}_{1}+\mathbf{e}_{2}}^{\mathbf{u}} \\
& \Longleftrightarrow B_{x, x+\mathbf{e}_{1}}^{\mathbf{u}} \leqslant B_{x, x+\mathbf{e}_{2}}^{\mathbf{u}} \\
& \Longleftrightarrow \mathbf{b}_{1}^{\mathbf{u}, x}=x+\mathbf{e}_{1} \text {. }
\end{aligned}
$$

The third equivalence used additivity. A similar argument shows that $\mathbf{b}_{1}^{\mathbf{u}, x}=x+\mathbf{e}_{2}$ if and only if $\mathbf{b}_{1}^{*, \mathbf{u}, x+\mathbf{e}^{*}}=x+\mathbf{e}^{*}-\mathbf{e}_{2}$.

Lemma 4.5. Fix $\mathbf{u} \in$ ri $\mathcal{U}$. The process of arrows $\left\{\mathbf{b}_{1}^{\mathbf{u}, x}-x\right\}_{x \in \mathbb{Z}^{2}}$ is equal in distribution to the process $\left\{-x-\mathbf{e}^{*}-\mathbf{b}_{1}^{*, \mathbf{u},-x-\mathbf{e}^{*}}\right\}_{x \in \mathbb{Z}^{2}}$ of reversed dual arrows reflected across the origin.

Proof. Utilize again the process defined in (4.16). As observed, $\widetilde{B}^{\mathbf{u}}$ is the Busemann function in direction $\mathbf{u}$ of the LPP process (1.1) with weights $\tilde{Y}^{\mathbf{u}}$. In particular then processes $\widetilde{B}^{\mathbf{u}}$ and $B^{\mathbf{u}}$ are equal in distribution. Distributional equality $\left\{-x-\mathbf{b}_{1}^{\mathrm{sw}, \mathbf{u},-x}\right\}_{x \in \mathbb{Z}^{2}} \stackrel{d}{=}\left\{\mathbf{b}_{1}^{\mathbf{u}, x}-x\right\}_{x \in \mathbb{Z}^{2}}$ follows from these equivalences:

$$
\begin{aligned}
-x-\mathbf{b}_{1}^{\mathrm{sw}, \mathbf{u},-x}=\mathbf{e}_{1} & \Longleftrightarrow \mathbf{b}_{1}^{\mathrm{sw}, \mathbf{u},-x}=-x-\mathbf{e}_{1} \Longleftrightarrow B_{-x-\mathbf{e}_{1},-x}^{\mathbf{u}} \leqslant B_{-x-\mathbf{e}_{2},-x}^{\mathbf{u}} \\
& \Longleftrightarrow \widetilde{B}_{x, x+\mathbf{e}_{1}}^{\mathbf{u}} \leqslant \widetilde{B}_{x, x+\mathbf{e}_{2}}^{\mathbf{u}}
\end{aligned}
$$

and

$$
\mathbf{b}_{1}^{\mathbf{u}, x}-x=\mathbf{e}_{1} \Longleftrightarrow B_{x, x+\mathbf{e}_{1}}^{\mathbf{u}} \leqslant B_{x, x+\mathbf{e}_{2}}^{\mathbf{u}} .
$$

The claim of the lemma follows from $-x-\mathbf{e}^{*}-\mathbf{b}_{1}^{*, \mathbf{u},-x-\mathbf{e}^{*}}=-x-\mathbf{b}_{1}^{\mathrm{sw}, \mathbf{u},-x}$.

The message of the last two lemmas is that the up-right directed $B^{\mathbf{u}}$-geodesics $\left\{\mathbf{b}^{\mathbf{u}, x}: x \in \mathbb{Z}^{2}\right\}$ and the down-left directed dual $B^{\mathbf{u}}$-geodesics $\left\{\mathbf{b}^{*, \mathbf{u}, z}: z \in \mathbb{Z}^{2 *}\right\}$ never cross each other but are equal in distribution, modulo a shift by $\mathbf{e}^{*}$ and a lattice reflection across the origin. 
4.3. Coalescence and the bi-infinite geodesic. The backward $B^{\mathbf{u}}$-cluster $\mathcal{C}^{\mathbf{u}}(x)$ at $x$ consists of those points $y$ whose $B^{\mathbf{u}}$-geodesic goes through $x$ :

$$
\mathcal{C}^{\mathbf{u}}(x)=\left\{y \in x+\mathbb{Z}_{\leqslant 0}^{2}: \mathbf{b}_{|x-y|_{1}}^{\mathbf{u}, y}=x\right\} .
$$

A bi-infinite up-right nearest-neighbor path $\left\{x_{k}\right\}_{k \in \mathbb{Z}}$ on $\mathbb{Z}^{2}$ is a bi-infinite $B^{\mathbf{u}}$-geodesic if $\mathbf{b}_{\ell-k}^{\mathbf{u}, x_{k}}=x_{\ell}$ for all indices $k<\ell$ in $\mathbb{Z}$. If two $B^{\mathbf{u}}$-geodesics $\mathbf{b}^{\mathbf{u}, x}$ and $\mathbf{b}^{\mathbf{u}, y}$ have a point in common they coalesce: namely, if $\mathbf{b}_{m}^{\mathbf{u}, x}=\mathbf{b}_{n}^{\mathbf{u}, y}$ then $\mathbf{b}_{m+k}^{\mathbf{u}, x}=\mathbf{b}_{n+k}^{\mathbf{u}, y}$ for all $k \geqslant 0$.

Consider the following three events.

(i) $\quad$ there exists a bi-infinite $B^{\mathbf{u}}$-geodesic $\}$

(ii) $\left\{\exists x \in \mathbb{Z}^{2}\right.$ such that $\mathcal{C}^{\mathbf{u}}(x)$ is infinite $\}$

(iii) $\left\{\exists x, y \in \mathbb{Z}^{2}\right.$ such that the $B^{\mathbf{u}}$-geodesics $\mathbf{b}^{\mathbf{u}, x}$ and $\mathbf{b}^{\mathbf{u}, y}$ are disjoint $\}$.

The goal is to show that almost surely none of these happen. The first step is to show that they happen together, modulo the duality.

Lemma 4.6. Fix $\mathbf{u} \in \mathrm{ri \mathcal {U }}$. Then all three events in (4.19) have equal probability.

Proof. Step 1. $\{(\mathrm{i})\}=\{($ ii $)\}$. For one direction, any point on a bi-infinite $B^{\mathbf{u}}$-geodesic has an infinite backward $B^{\mathbf{u}}$-cluster. Conversely, suppose $\mathcal{C}^{\mathbf{u}}\left(x_{0}\right)$ is infinite. Then for each $m \in \mathbb{Z}_{>0}$ there exists $y(m) \in x_{0}+\mathbb{Z}_{\leqslant 0}^{2}$ such that $\mathbf{b}_{m}^{\mathbf{u}, y(m)}=x_{0}$. From the finite paths $\mathbf{b}_{0, m}^{\mathbf{u}, y(m)}$, a compactness argument produces an infinite backward path $\left\{x_{i}\right\}_{i \leqslant 0}$ such that $\mathbf{b}_{1}^{\mathbf{u}, x_{i}}=x_{i+1}$ for all $i<0$. Extend this infinite backward path to a bi-infinite $B^{\mathbf{u}_{\text {-geodesic }}} x_{\text {. by defining }} x_{i}=\mathbf{b}_{i}^{\mathbf{u}, x_{0}}$ for $i>0$.

Here is the compactness argument. Choose nested subsequences of indices $\left\{m_{j}^{1}\right\}_{j \geqslant 1} \supset\left\{m_{j}^{2}\right\}_{j \geqslant 1} \supset$ $\cdots \supset\left\{m_{j}^{k}\right\}_{j \geqslant 1} \supset \cdots$ such that, for each $k, m_{1}^{k} \geqslant k$ and the $k$-step path segments $\mathbf{b}_{m_{j}^{k}-k, m_{j}^{k}}^{\mathbf{u}, y\left(m_{j}^{k}\right)}$ converge to a path $x_{-k, 0}$ as $j \rightarrow \infty$. Convergent subsequences exist because the $k$-step segments $\left\{\mathbf{b}_{m-k, m}^{\mathbf{u}, y(m)}\right\}_{m \geqslant k}$ lie in the finite set of $k$-step paths that end at $x_{0}$. Since the subsequences are nested, the limits are consistent and form a single backward nearest-neighbor path $\left\{x_{i}\right\}_{i \leqslant 0}$. Since the convergence happens on a discrete set, for each $k$ there exists $j(k)<\infty$ such that $\mathbf{b}_{m_{j}^{k}-k, m_{j}^{k}}^{\mathbf{u}, y\left(m_{j}^{k}\right)}=x_{-k, 0}$ for $j \geqslant j(k)$. This implies that $\mathbf{b}_{1}^{\mathbf{u}, x_{i}}=x_{i+1}$ for all $i<0$.

Step 2. $\mathbb{P}\{($ iii $)\} \leqslant \mathbb{P}\{($ ii $)\}$. Suppose event (iii) happens and let points $x_{0}, y_{0} \in \mathbb{Z}^{2}$ be such that geodesics $\mathbf{b}^{\mathbf{u}, x_{0}}$ and $\mathbf{b}^{\mathbf{u}, y_{0}}$ are disjoint. By the limit in (4.4), both coordinates $\mathbf{b}_{n}^{\mathbf{u}, x_{0}} \cdot \mathbf{e}_{1}$ and $\mathbf{b}_{n}^{\mathbf{u}, x_{0}} \cdot \mathbf{e}_{2}$ increase to $\infty$ as $n \rightarrow \infty$, and the same for $\mathbf{b}^{\mathbf{u}, y_{0}}$. By suitably redefining the initial points we can assume that $x_{0}$ and $y_{0}$ lie on the same antiodiagonal (that is, $x_{0} \cdot\left(\mathbf{e}_{1}+\mathbf{e}_{2}\right)=y_{0} \cdot\left(\mathbf{e}_{1}+\mathbf{e}_{2}\right)$ ) and $x_{0} \cdot \mathbf{e}_{2}<y_{0} \cdot \mathbf{e}_{2}$, so that $\mathbf{b}^{\mathbf{u}, y_{0}}$ is above and to the left of $\mathbf{b}^{\mathbf{u}, x_{0}}$.

Let us say that a dual point $z \in \mathbb{Z}^{2 *}$ lies between the two geodesics if the ray $\left\{z+t\left(\mathbf{e}_{2}-\mathbf{e}_{1}\right): t \geqslant 0\right\}$ hits a point of $\mathbf{b}^{\mathbf{u}, y_{0}}$ and the ray $\left\{z+t\left(\mathbf{e}_{1}-\mathbf{e}_{2}\right): t \geqslant 0\right\}$ hits a point of $\mathbf{b}^{\mathbf{u}, x_{0}}$.

For each $z \in \mathbb{Z}^{2 *}$ that lies between the two geodesics, at least one of $z+\mathbf{e}_{1}$ and $z+\mathbf{e}_{2}$ also lies between the two geodesics. For if $z+\mathbf{e}_{1}$ does not lie between the two geodesics, then edge $\left\{z, z+\mathbf{e}_{1}\right\}$ must cross an edge of $\mathbf{b}^{\mathbf{u}, x_{0}}$, and this edge is $\left\{z+\left(\frac{1}{2},-\frac{1}{2}\right), z+\left(\frac{1}{2}, \frac{1}{2}\right)\right\}$. Similarly, if $z+\mathbf{e}_{2}$ does not lie between the two geodesics, the edge $\left\{z+\left(-\frac{1}{2}, \frac{1}{2}\right), z+\left(\frac{1}{2}, \frac{1}{2}\right)\right\}$ belongs to $\mathbf{b}^{\mathbf{u}, y_{0}}$. Thus if neither $z+\mathbf{e}_{1}$ nor $z+\mathbf{e}_{2}$ lies between the two geodesics, the two geodesics meet at the point $z+\left(\frac{1}{2}, \frac{1}{2}\right)$, contrary to the assumption of no coalescence.

Thus we can choose a semi-infinite path $\left\{z_{m}\right\}_{m \in \mathbb{Z}_{\geqslant 0}}$ on the dual lattice such that $z_{0} \cdot\left(\mathbf{e}_{1}+\mathbf{e}_{2}\right)=$ $y_{0} \cdot\left(\mathbf{e}_{1}+\mathbf{e}_{2}\right), z_{m+1} \in\left\{z_{m}+\mathbf{e}_{1}, z_{m}+\mathbf{e}_{2}\right\}$ for all $m$, and the entire path $z_{\bullet}$ lies between the geodesics $\mathbf{b}^{\mathbf{u}, x_{0}}$ and $\mathbf{b}^{\mathbf{u}, y_{0}}$. Since $B^{\mathbf{u}}$-geodesics and dual $B^{\mathbf{u}}$-geodesics never cross, the (finite) dual geodesics $\mathbf{b}_{0, m}^{*, \mathbf{u}, z_{m}}$ must also lie between the geodesics $\mathbf{b}^{\mathbf{u}, x_{0}}$ and $\mathbf{b}^{\mathbf{u}, y_{0}}$. In particular, the endpoints $\left\{\mathbf{b}_{m}^{*, \mathbf{u}, z_{m}}\right\}_{m \in \mathbb{Z}_{\geqslant 0}}$ lie on the bounded antidiagonal segment between $x_{0}$ and $y_{0}$. By compactness there is a subsequence $z_{m_{j}}$ 
such that the endpoint converges: $\mathbf{b}_{m_{j}}^{*, \mathbf{u}, z_{m_{j}}} \rightarrow z^{*}$. Since this convergence happens on a discrete set, there exists some $j_{0}$ such that $\mathbf{b}_{m_{j}}^{*, \mathbf{u}, z_{m_{j}}}=z^{*}$ for all $j \geqslant j_{0}$. Thereby the (dual) backward $B^{\mathbf{u}}$-cluster $\mathcal{C}^{*, \mathbf{u}}\left(z^{*}\right)$ is infinite.

We have shown that event (iii) implies that event (ii) happens for dual geodesics. By the distribu-

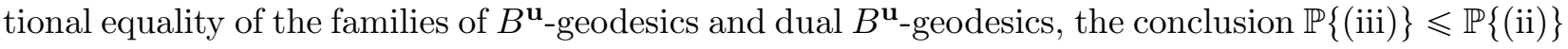
follows.

Step 3. $\mathbb{P}\{(\mathrm{i})\} \leqslant \mathbb{P}\{($ iii $)\}$. Let $x$ and $y$ be two points on $\mathbb{Z}^{2}$ on opposite sides of a bi-infinite dual $B^{\mathbf{u}}$-geodesic. Geodesics $\mathbf{b}^{\mathbf{u}, x}$ and $\mathbf{b}^{\mathbf{u}, y}$ cannot cross the dual $B^{\mathbf{u}}$-geodesic (Lemma 4.4), and hence cannot coalesce.

TheOREM 4.7. Fix $\mathbf{u} \in \mathrm{ri \mathcal {U }}$. Then all three events in (4.19) have zero probability.

Proof. This theorem follows from Lemma 4.6 and

$\mathbb{P}\left\{\right.$ there exists a bi-infinite $B^{\mathbf{u}}$-geodesic $\}=0$.

To prove (4.20) we use the solution of the midpoint problem to prove that a bi-infinite $B^{\mathbf{u}}$-geodesic goes through the origin with probability zero. Suppose $\left\{x_{n}\right\}_{n \in \mathbb{Z}}$ is a bi-infinite $B^{\mathbf{u}}$-geodesic with $x_{0}=0$. To apply Theorem 3.6 to $u_{n}=x_{-n}, z_{n}=0$ and $v_{n}=x_{n}$ we need the limits

$$
\frac{x_{-n}}{n} \rightarrow-\mathbf{u} \text { and } \frac{x_{n}}{n} \rightarrow \mathbf{u}
$$

almost surely on the event where a bi-infinite $B^{\mathbf{u}}$-geodesic through the origin exists.

The second limit of (4.21) is in (4.4). The backward limit $x_{-n} / n \rightarrow-\mathbf{u}$ is proved by the same argument. Namely, since $x_{-n, 0}$ is a (finite) $B^{\mathbf{u}_{-g e o d e s i c}}$ (that is, $\mathbf{b}_{j}^{\mathbf{u}, x_{-n}}=x_{-n+j}$ for $0 \leqslant j \leqslant n$ ), (4.3) applies and gives

$$
G_{x_{-n}, 0}=B_{x_{-n}, 0}^{\mathbf{u}}+Y_{0} .
$$

The uniform passage time limit (1.4) applies to the southwest LPP process to give

$$
G_{x_{-n}, 0}=g\left(-x_{-n}\right)+o(n) \quad \text { almost surely. }
$$

The uniform ergodic theorem for cocycles (Theorem B.1) gives

$$
B_{x_{-n}, 0}^{\mathbf{u}}=\nabla g(\mathbf{u}) \cdot\left(-x_{-n}\right)+o(n) \quad \text { almost surely. }
$$

These almost sure asymptotics and strict concavity of $g$ in the form (4.6) then imply that the first limit in (4.21) holds almost surely on the event where a bi-infinite $B^{\mathbf{u}}$-geodesic $x$. through $x_{0}=0$ exists.

Since $x_{-n, n}$ is a geodesic through the origin, we have $0 \in \pi^{x_{-n}, x_{n}}$ for all $n>0$ on the event where the bi-infinite geodesic $x$. goes through the origin. By Theorem 3.6 this event must have probability zero.

\subsection{Completion of the proofs.}

Proof of Theorem 2.2. Lemma 4.1(iii) implies that almost surely there is a unique u-directed semiinfinite geodesic out of $x$, namely the $B^{\mathbf{u}}$-geodesic $\mathbf{b}^{\mathbf{u}, x}$. Its construction (4.1) shows that it is a Borel function of the random variables $B_{x, y}^{\mathbf{u}}$ which in turn are Borel functions of $Y$. Theorem 4.7 gives the almost sure coalescence and non-existence of a bi-infinite geodesic. (A bi-infinite $\mathbf{u}$-directed geodesic $\left(x_{i}\right)_{i \in \mathbb{Z}}$ must also be a $B^{\mathbf{u}}$-geodesic because by Lemma 4.1 (iii), $\left(x_{i}\right)_{i \geqslant \ell}=\mathbf{b}^{\mathbf{u}, x_{\ell}}$ for each $\ell \in \mathbb{Z}$.)

Proof of Theorem 2.3. Process $\tilde{Y}^{\mathbf{u}}$ was defined in (4.16) and hence by (3.19) satisfies

$$
\tilde{Y}_{x}^{\mathbf{u}}=X_{-x}^{\mathbf{u}}=B_{-x-\mathbf{e}_{1},-x}^{\mathbf{u}} \wedge B_{-x-\mathbf{e}_{2},-x}^{\mathbf{u}} .
$$

The i.i.d. $\operatorname{Exp}(1)$ distribution of $X^{\mathbf{u}}$ gives the same to $\tilde{Y}^{\mathbf{u}}$, limit (3.16) shows that $\tilde{Y}^{\mathbf{u}}$ is a Borel function of $Y$, and the second equality of the display above implies that $\tilde{Y}_{x}^{\mathbf{u}}\left(\theta_{y} \omega\right)=\tilde{Y}_{x-y}^{\mathbf{u}}(\omega)$. 
Lemma 4.4 and definition (2.3) imply that $\mathcal{T}_{\mathbf{u}}^{*}=\bigcup_{z \in \mathbb{Z}^{2 *}} \mathbf{b}^{*, \mathbf{u}, z}$. Tracing through definition (2.4) of $\widetilde{\mathcal{T}}_{\mathbf{u}}$, definition (4.17) of dual geodesics, and (4.18) gives the equivalence

$$
\left\{x, x+\mathbf{e}_{1}\right\} \in \widetilde{\mathcal{T}}_{\mathbf{u}} \Longleftrightarrow \widetilde{B}_{x, x+\mathbf{e}_{1}}^{\mathbf{u}} \leqslant \widetilde{B}_{x, x+\mathbf{e}_{2}}^{\mathbf{u}} .
$$

A similar argument gives $\left\{x, x+\mathbf{e}_{2}\right\} \in \widetilde{\mathcal{T}}_{\mathbf{u}} \Longleftrightarrow \widetilde{B}_{x, x+\mathbf{e}_{1}}^{\mathbf{u}}>\widetilde{B}_{x, x+\mathbf{e}_{2}}^{\mathbf{u}}$. The proof of Lemma 4.5 observed that $\widetilde{B}^{\mathbf{u}}$ is the Busemann function of the LPP process with weights $\tilde{Y}^{\mathbf{u}}$. Hence (4.5) applied to weights $\tilde{Y}^{\mathbf{u}}$ implies that $\widetilde{\mathcal{T}}_{\mathbf{u}}$ is the tree of semi-infinite $\mathbf{u}$-directed geodesics for this LPP process.

Proof of Theorem 2.4. Define $a_{j} \in\{1,2\}$ by

$$
a_{j}= \begin{cases}1, & B_{(N+j,-j-1),(N+j+1,-j-1)}^{\mathbf{u}} \leqslant B_{(N+j,-j-1),(N+j,-j)}^{\mathbf{u}} \\ 2, & B_{(N+j,-j-1),(N+j,-j)}^{\mathbf{u}}<B_{(N+j,-j-1),(N+j+1,-j-1)}^{\mathbf{u}} .\end{cases}
$$

Property (a) in Definition 3.1 applied to the down-right path

$$
y_{2 j}=(N+j,-j-1), \quad y_{2 j+1}=(N+j+1,-j-1)
$$

implies that $\left\{a_{j}\right\}_{j \in \mathbb{Z}}$ are i.i.d. random variables with marginal distribution

$$
\mathbb{P}\left(a_{j}=1\right)=\alpha=1-\mathbb{P}\left(a_{j}=2\right) .
$$

The process $\left\{\xi_{j}\right\}$ is obtained from the connection

$$
\xi_{j}=\left\{\begin{array}{l}
s \\
c \\
h \\
v
\end{array} \quad \text { if } \quad\left(a_{j-1}, a_{j}\right)=\left\{\begin{array}{l}
(2,1) \\
(1,2) \\
(1,1) \\
(2,2) .
\end{array}\right.\right.
$$

Thus $\left\{\xi_{j}\right\}$ has the distribution of the Markov chain $X_{j}=\left(a_{j-1}, a_{j}\right)$, after relabeling the states as above.

\section{INCREMENT-STATIONARY LPP AND COMPETITON INTERFACE}

This section explains how $B^{\mathbf{u}}$ represents a LPP process with boundary conditions and how the paths $\mathbf{b}^{\mathbf{u}, x}$ and $\mathbf{b}^{\mathrm{sw}, \mathbf{u}, x}$ function both as geodesics and competition interfaces, depending on whether the LPP uses weights $Y$ or $X^{\mathbf{u}}$. Fix $\mathbf{u} \in$ ri $\mathcal{U}$. Fix also a down-right path $\mathcal{Y}=\left(y_{k}\right)_{k \in \mathbb{Z}}$ on $\mathbb{Z}^{2}$, that is, a sequence in $\mathbb{Z}^{2}$ such that $y_{k}-y_{k-1} \in\left\{\mathbf{e}_{1},-\mathbf{e}_{2}\right\}$ for all $k \in \mathbb{Z}$. Let $\mathcal{H}^{ \pm}$be as in (3.1)-(3.2) and define $\widetilde{\mathcal{H}}^{ \pm}=\mathcal{Y} \cup \mathcal{H}^{ \pm}$. $\mathcal{Y}$ serves as a boundary and the LPP processes will be defined in the regions $\widetilde{\mathcal{H}}^{ \pm}$.

Let $|\pi|$ denote the Euclidean length (number of edges) of a nearest-neighbor lattice path. For $x \in \widetilde{\mathcal{H}}^{+}$, let $\Pi^{\mathcal{Y}, x}$ be the set of up-right paths $\pi=\pi_{0, n}=\left(\pi_{i}\right)_{i=0}^{n}$ of any length $n=|\pi|$ that go from $\mathcal{Y}$ to $x$ and that lie in $\mathcal{H}^{+}$except for the initial point on $\mathcal{Y}$ :

$$
\Pi^{\mathcal{Y}, x}=\left\{\pi: \pi \in \Pi_{\pi_{0}, x}, \pi_{0} \in \mathcal{Y}, \pi_{1,|\pi|} \subset \mathcal{H}^{+}\right\} .
$$

For $x \in \widetilde{\mathcal{H}}^{+}$define the LPP process

$$
H_{x}^{+}=\sup _{\pi \in \Pi^{\mathcal{Y}, x}}\left\{B_{y_{0}, \pi_{0}}^{\mathbf{u}}+\sum_{i=1}^{|\pi|} X_{\pi_{i}}^{\mathbf{u}}\right\} .
$$

In the degenerate case $x \in \mathcal{Y}$ and $H_{x}^{+}=B_{y_{0}, x}^{\mathbf{u}}$. The set of paths maximized over can be finite (for example in case $\lim _{k \rightarrow-\infty} y_{k} \cdot \mathbf{e}_{2}=\infty$ and $\lim _{k \rightarrow \infty} y_{k} \cdot \mathbf{e}_{1}=\infty$ ) or infinite (for example if $y_{k}=k \mathbf{e}_{1}$ is the $x$-axis). The random variables $X_{x}^{\mathbf{u}}$ over $x \in \mathcal{H}^{+}$and $B_{y_{k}, y_{k+1}}^{\mathbf{u}}$ on $\mathcal{Y}$ are all independent, so $H^{+}$is 
an LPP process that uses independent weights. To ensure unique geodesics, we restrict ourselves to the full-measure event on which

$$
\text { no two nonempty sums of distinct }\left\{X_{x}^{\mathbf{u}}\right\}_{x \in \mathcal{H}^{+}} \text {and }\left\{B_{y_{k}, y_{k+1}}^{\mathbf{u}}\right\}_{k \in \mathbb{Z}} \text { agree. }
$$

A combination of (3.19) and (4.11), as in the proof of Lemma 4.1(i), shows that the LPP process $H^{+}$coincides with $B^{\mathbf{u}}$ and that the southwest geodesics are the geodesics in this process.

Proposition 5.1. Fix $x \in \mathcal{H}^{+}$. Let $n=\min \left\{i \geqslant 0: \mathbf{b}_{i}^{\mathrm{sw}, \mathbf{u}, x} \in \mathcal{Y}\right\}$. Then $\left\{\pi_{i}^{+, x}=\mathbf{b}_{n-i}^{\mathrm{sw}, \mathbf{u}, x}\right\}_{0 \leqslant i \leqslant n}$ is the unique maximizing path in (5.1) and

$$
H_{x}^{+}=B_{y_{0}, \pi_{0}^{+, x}}^{\mathbf{u}}+\sum_{i=1}^{n} X_{\pi_{i}^{+, x}}^{\mathbf{u}}=B_{y_{0}, x}^{\mathbf{u}} .
$$

For $A \subset \mathcal{Y}$, let $\mathcal{H}_{A}^{+}=\left\{x \in \widetilde{\mathcal{H}}^{+}: \pi_{0}^{+, x} \in A\right\}$ denote the set of points $x$ whose geodesic emanates from $A$. Fix two adjacent points $y_{m}, y_{m+1}$ on $\mathcal{Y}$. Decompose $\mathcal{H}^{+}=\mathcal{H}_{y_{-\infty}, m}^{+} \cup \mathcal{H}_{y_{m+1, \infty}}^{+}$according to whether the geodesic emanates from $\left\{y_{k}\right\}_{k \leqslant m}$ or $\left\{y_{k}\right\}_{k \geqslant m+1}$. The two regions $\mathcal{H}_{y_{-\infty, m}}^{+}$and $\mathcal{H}_{y_{m+1, \infty}}^{+}$ are separated by an up-right path $\varphi^{+}=\left(\varphi_{n}^{+}\right)_{n \geqslant 0}$ called the competition interface:

$$
\begin{aligned}
\varphi_{0}^{+} & = \begin{cases}y_{m}, & B_{y_{0}, y_{m}}^{\mathbf{u}}<B_{y_{0}, y_{m+1}}^{\mathbf{u}} \\
y_{m+1}, & B_{y_{0}, y_{m+1}}^{\mathbf{u}}<B_{y_{0}, y_{m}}^{\mathbf{u}}\end{cases} \\
\text { and for } k \geqslant 0 \quad \varphi_{k+1}^{+} & = \begin{cases}\varphi_{k}^{+}+\mathbf{e}_{1}, & H_{\varphi_{k}^{+}+\mathbf{e}_{1}}^{+}<H_{\varphi_{k}^{+}+\mathbf{e}_{2}}^{+} \\
\varphi_{k}^{+}+\mathbf{e}_{2}, & H_{\varphi_{k}^{+}+\mathbf{e}_{2}}^{+}<H_{\varphi_{k}^{+}+\mathbf{e}_{1}}^{+}\end{cases}
\end{aligned}
$$

One can check inductively that for each $n \in \mathbb{Z}_{\geqslant 0}, \varphi_{n}^{+}$is the unique point on its antidiagonal $\{x \in$ $\left.\widetilde{\mathcal{H}}^{+}: x \cdot\left(\mathbf{e}_{1}+\mathbf{e}_{2}\right)=\varphi_{0}^{+} \cdot\left(\mathbf{e}_{1}+\mathbf{e}_{2}\right)+n\right\}$ that satisfies $\varphi_{n}^{+}+\mathbb{Z}_{>0} \mathbf{e}_{2} \subset \mathcal{H}_{y_{-\infty}, m}^{+}$and $\varphi_{n}^{+}+\mathbb{Z}_{>0} \mathbf{e}_{1} \subset \mathcal{H}_{y_{m+1, \infty}}^{+}$. Comparison of (4.1) and (5.4), with an appeal to (5.3), proves the next characterization of $\varphi^{+}$.

Proposition 5.2. $\varphi^{+}=\mathbf{b}^{\mathbf{u}, \varphi_{0}^{+}}$.

An analogous LPP process is defined for $x \in \widetilde{\mathcal{H}}^{-}$with weights $Y$ :

$$
H_{x}^{-}=\sup _{\pi \in \Pi^{x, \mathcal{Y}}}\left\{\sum_{i=0}^{|\pi|-1} Y_{\pi_{i}}+B_{\pi_{|\pi|}, y_{0}}^{\mathbf{u}}\right\}
$$

where $\Pi^{x, \mathcal{Y}}$ is the set of up-right paths from $x$ to $\mathcal{Y}$ that lie in $\mathcal{H}^{-}$except for their final point on $\mathcal{Y}$. This time $H_{x}^{-}=B_{x, y_{0}}^{\mathbf{u}}$, the part of $\mathbf{b}^{\mathbf{u}, x}$ between $x$ and $\mathcal{Y}$ is the geodesic, and competition interfaces are southwest geodesics $\mathbf{b}^{\mathrm{sw}, \mathbf{u}, y}$ emanating from points $y \in \mathcal{Y}$. We omit the details.

From the results of this section we can derive Lemma 4.4 of [3] as a special case. Namely, fix $(m, n) \in \mathbb{Z}_{>0}^{2}$ and take $\mathcal{H}^{-}$to be the southwest quadrant bounded on the north and east by the path $y_{k}^{-}=(m, n)-k^{+} \mathbf{e}_{2}-k^{-} \mathbf{e}_{1}$. In terms of the LPP process $H^{-}$and the weights $Y$ in (5.5) above, the "reversed process" $G^{*}$ and weights $\omega^{*}$ in [3] correspond to $G_{i j}^{*}=H_{(m-i, n-j)}^{-}$and $\omega_{i j}^{*}=Y_{(m-i, n-j)}$. The competition interface that emanates from the "origin" $(m, n)$ for $H^{-}$in $(5.5)$ is the southwest geodesic $\mathbf{b}^{\mathrm{sw}, \mathbf{u},(m, n)}$. According to Proposition 5.1 above this path is also the geodesic for LPP process $H^{+}$constructed with coordinate axes boundary $y_{k}=k^{+} \mathbf{e}_{1}+k^{-} \mathbf{e}_{2}$. This is exactly what Lemma 4.4 of [3] says.

\section{Appendix A. Planar monotonicity}

Planar LPP increments possess monotonicity properties. The lemma below can be found proved as Lemma 4.6 in [19]. Let the LPP process $G$ be defined by (1.1) and define increments

$$
I_{x, v}=G_{x, v}-G_{x+e_{1}, v} \quad \text { and } \quad J_{y, v}=G_{y, v}-G_{y+e_{2}, v} .
$$


Lemma A.1. For $x, y, v \in \mathbb{Z}^{2}$ such that $x \leqslant v-e_{1}$ and $y \leqslant v-e_{2}$

$$
I_{x, v+e_{2}} \geqslant I_{x, v} \geqslant I_{x, v+e_{1}} \quad \text { and } \quad J_{y, v+e_{2}} \leqslant J_{y, v} \leqslant J_{y, v+e_{1}} .
$$

\section{Appendix B. Cocycle ergodic theorem}

Recall Definition 3.2. Covariant integrable cocycles satisfy a uniform ergodic theorem, sometimes also called a shape theorem.

Theorem B.1. Let $F \in \mathcal{K}$ be such that $\mathbb{E}[F(x, y)]=0 \forall x, y \in \mathbb{Z}^{2}$. Assume that there exists a function $\bar{F}: \Omega \times\left\{\mathbf{e}_{1}, \mathbf{e}_{2}\right\} \rightarrow \mathbb{R}$ such that, $\mathbb{P}$-almost surely and for $k \in\{1,2\}, F\left(\omega, 0, \mathbf{e}_{k}\right) \leqslant \bar{F}\left(\omega, \mathbf{e}_{k}\right)$ and

$$
\varlimsup_{\delta \searrow 0} \varlimsup_{n \rightarrow \infty} \max _{|x|_{1} \leqslant n} \frac{1}{n} \sum_{0 \leqslant i \leqslant n \delta}\left|\bar{F}\left(\theta_{x+i \mathbf{e}_{k}} \omega, \mathbf{e}_{k}\right)\right|=0
$$

Then

$$
\lim _{n \rightarrow \infty} \max _{|x|_{1} \leqslant n} \frac{|F(\omega, 0, x)|}{n}=0 \quad \mathbb{P} \text {-a.s. }
$$

For a proof see Appendix A.3 of [12]. A sufficient condition for limit (B.1) is that $\mathbb{E}\left|\bar{F}\left(\omega, \mathbf{e}_{k}\right)\right|^{2+\varepsilon}<$ $\infty$ for some $\varepsilon>0$ and the shifts of $\bar{F}$ have finite range of dependence: namely, $\exists r_{0}<\infty$ such that if $\left|x_{i}-x_{j}\right| \geqslant r_{0}$ for each pair $i \neq j$, then $\left\{\left(\bar{F}\left(\theta_{x_{i}} \omega, \mathbf{e}_{k}\right)\right)_{k \in\{1,2\}}: 1 \leqslant i \leqslant m\right\}$ is a sequence of $m$ independent random vectors.

\section{REFERENCES}

[1] Yuri Bakhtin, Eric Cator, and Konstantin Khanin. Space-time stationary solutions for the Burgers equation. J. Amer. Math. Soc., 27(1):193-238, 2014.

[2] Yuri Bakhtin and Liying Li. Thermodynamic limit for directed polymers and stationary solutions of the burgers equation. Communications on Pure and Applied Mathematics, 2018. To appear.

[3] Márton Balázs, Eric Cator, and Timo Seppäläinen. Cube root fluctuations for the corner growth model associated to the exclusion process. Electron. J. Probab., 11:no. 42, 1094-1132 (electronic), 2006.

[4] Eric Cator and Leandro P. R. Pimentel. A shape theorem and semi-infinite geodesics for the Hammersley model with random weights. ALEA Lat. Am. J. Probab. Math. Stat., 8:163-175, 2011.

[5] Jon Chaika and Arjun Krishnan. Stationary coalescing walks on the lattice. 2016. To appear in Probab. Theory Rel. Fields. arXiv: 1612.00434.

[6] Michael Damron and Jack Hanson. Busemann functions and infinite geodesics in two-dimensional firstpassage percolation. Comm. Math. Phys., 325(3):917-963, 2014.

[7] Michael Damron and Jack Hanson. Bigeodesics in first-passage percolation. Comm. Math. Phys., 349(2):753-776, 2017.

[8] Elnur Emrah. Limit shapes for inhomogeneous corner growth models with exponential and geometric weights. Electron. Commun. Probab., 21:Paper No. 42, 16, 2016.

[9] Wai-Tong (Louis) Fan and Timo Seppäläinen. Joint distribution of Busemann functions in the exactly solvable corner growth model. 2018. arXiv:1808.09069.

[10] Pablo A. Ferrari and Leandro P. R. Pimentel. Competition interfaces and second class particles. Ann. Probab., 33(4):1235-1254, 2005.

[11] Nicos Georgiou, Firas Rassoul-Agha, and Timo Seppäläinen. Geodesics and the competition interface for the corner growth model. Probab. Theory Related Fields, 169(1-2):223-255, 2017.

[12] Nicos Georgiou, Firas Rassoul-Agha, Timo Seppäläinen, and Atilla Yilmaz. Ratios of partition functions for the log-gamma polymer. Ann. Probab., 43(5):2282-2331, 2015.

[13] C. Douglas Howard and Charles M. Newman. Geodesics and spanning trees for Euclidean first-passage percolation. Ann. Probab., 29(2):577-623, 2001.

[14] Cristina Licea and Charles M. Newman. Geodesics in two-dimensional first-passage percolation. Ann. Probab., 24(1):399-410, 1996.

[15] James B. Martin. Limiting shape for directed percolation models. Ann. Probab., 32(4):2908-2937, 2004. 
[16] Charles M. Newman. A surface view of first-passage percolation. In Proceedings of the International Congress of Mathematicians, Vol. 1, 2 (Zürich, 1994), pages 1017-1023, Basel, 1995. Birkhäuser.

[17] Leandro P. R. Pimentel. Duality between coalescence times and exit points in last-passage percolation models. Ann. Probab., 44(5):3187-3206, 2016.

[18] Hermann Rost. Nonequilibrium behaviour of a many particle process: density profile and local equilibria. Z. Wahrsch. Verw. Gebiete, 58(1):41-53, 1981.

[19] Timo Seppäläinen. The corner growth model with exponential weights. In Random growth models, volume 75 of Proc. Sympos. Appl. Math., pages 133-201. Amer. Math. Soc., Providence, RI, 2018. arXiv: 1709.05771.

Timo Seppëläinen, University of Wisconsin-Madison, Mathematics Department, Van Vleck Hall, 480 Lincoln Dr., MADison WI 53706-1388, USA.

E-mail address: seppalai@math.wisc.edu

URL: http://www.math.wisc.edu/ seppalai 